\title{
Chapitre I - Lieu ecclésial et agencement du décor sculpté
}

Jérôme Baschet, Jean-Claude Bonne et Pierre-Olivier Dittmar

\section{(2) OpenEdition}

\section{Journals}

Édition électronique

URL : http://journals.openedition.org/imagesrevues/1608

DOI : 10.4000/imagesrevues. 1608

ISSN : 1778-3801

Éditeur :

Centre d'Histoire et Théorie des Arts, Groupe d'Anthropologie Historique de l'Occident Médiéval, Laboratoire d'Anthropologie Sociale, UMR 8210 Anthropologie et Histoire des Mondes Antiques

\section{Référence électronique}

Jérôme Baschet, Jean-Claude Bonne et Pierre-Olivier Dittmar, « Chapitre I - Lieu ecclésial et agencement du décor sculpté », Images Re-vues [En ligne], Hors-série 3 | 2012, mis en ligne le 24 novembre 2012, consulté le 30 janvier 2021. URL : http://journals.openedition.org/imagesrevues/1608 ; DOI : https://doi.org/10.4000/imagesrevues.1608

Ce document a été généré automatiquement le 30 janvier 2021.

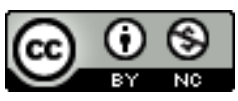

Images Re-vues est mise à disposition selon les termes de la Licence Creative Commons Attribution -

Pas d'Utilisation Commerciale 4.0 International. 


\title{
Chapitre I - Lieu ecclésial et agencement du décor sculpté
}

\author{
Jérôme Baschet, Jean-Claude Bonne et Pierre-Olivier Dittmar
}

1 Les images médiévales peuvent être considérées comme des images-objets et celles dont nous traitons ici, les chapiteaux des églises romanes auvergnates, comme des images-lieux ${ }^{1}$. Des images en situation, c'est-à-dire d'abord des images localisées, inscrites dans ce lieu bien particulier qu'est l'édifice ecclésial. Il s'avère donc indispensable de s'interroger sur la façon dont les chapiteaux s'agencent au sein du

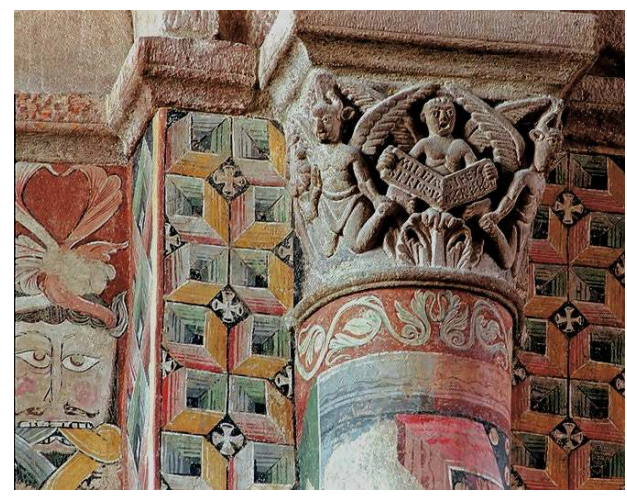
lieu et sur les interactions complexes entre ces images-objets et le fonctionnement de l'édifice cultuel lui-même. Cela suppose, tout d'abord, quelques remarques sur le statut de l'église au cours du Moyen Age central.

\section{L'édifice ecclésial, lieu d'images}

2 Les églises sont alors bien plus que de simples lieux de culte. Ce sont des lieux essentiels pour l'ensemble de l'organisation sociale. Les grands centres monastiques et les sanctuaires dotés de reliques attirant d'importants pèlerinages le sont de longue date; mais l'ensemble des édifices ecclésiaux le deviennent plus nettement encore, en lien avec les processus de spatialisation des rapports sociaux dont les $\mathrm{XI}^{\mathrm{e}}$ et $\mathrm{XII}^{\mathrm{e}}$ siècles constituent le moment le plus intense. La société connaît alors une réorganisation majeure à laquelle Robert Fossier a donné le nom d' « encellulement » : la fixation et le regroupement de l'habitat aboutit, dans la plupart des régions d'Occident, à la constitution d'un réseau stable de villages, organisés le plus souvent autour de l'église 
et du cimetière environnant (et même dans les régions où l'habitat reste dispersé, le pôle ecclésial est déterminant, en raison de la généralisation du réseau paroissial) ${ }^{2}$. Celui-ci devient un cadre de vie décisif, auquel s'attache la triple obligation du baptême, du versement de la dîme et de la sépulture. Des lors, les vivants sont regroupés autour de pôles constitués par les bâtiments ecclésiaux et les cimetières. Ou, pour mieux dire, ils se trouvent fixés dans des entités locales dont le binôme égliseterre des morts constitue le noyau central. Cette fixation spatiale constitue un élément déterminant de la dynamique des rapports sociaux, en particulier en ce qui concerne l'exercice de la domination seigneuriale.

3 Comme l'a souligné Alain Guerreau, « dans l'Europe féodale, l'espace n'était pas conçu comme continu et homogène, mais comme discontinu, hétérogène et polarisé $»^{3}$. Or, ce sont pour l'essentiel des bâtiments ecclésiaux qui assurent cette polarisation: l'aristocratie laïque et le château interviennent aussi, mais le contraste entre l'instabilité du réseau castral, marqué par des abandons et des changements de site fréquents, et la permanence du réseau ecclésial, indique bien lequel des deux pèse le plus lourd. Le cimetière, qui entoure désormais l'église et se retrouve par conséquent au cœur des espaces habités, joue également un rôle décisif. Une telle disposition est l'aboutissement d'une mutation considérable ${ }^{4}$. En effet, l'Antiquité romaine enterrait ses morts loin des zones habitées et le haut Moyen Age a connu des usages très divers : transfert des corps saints dans les églises urbaines, nécropoles en pleine campagne, sépultures isolées encore fréquentes à l'époque carolingienne. Puis, au XI ${ }^{e}$ siècle, le cimetière attenant à l'église, faisant l'objet d'un rituel spécifique de consécration, se généralise partout comme l'unique lieu autorisé de sépulture des défunts (avec l'église elle-même, et à l'exception des non baptisés et des excommuniés, qui en sont exclus). On assiste ainsi au regroupement des morts au cœur de l'habitat, rural ou urbain; autrement dit, c'est autour des morts que les vivants sont désormais rassemblés. Se rendre à l'église signifie alors traverser la terre des morts ou, si l'on se rappelle la présence des reliques et des sépultures privilégiées dans le sol du bâtiment, c'est véritablement pénétrer dans le monde des morts.

Récemment mise en lumière, l'émergence d'une nouvelle doctrine du lieu de culte n'est pas étrangère à l'importance accrue que les édifices ecclésiaux acquièrent dans le fonctionnement de la société du temps ${ }^{5}$. Les chrétiens de l'Antiquité tardive et des premiers siècles médiévaux concevaient la communauté sur un mode essentiellement spirituel et tendaient à minimiser l'importance du lieu matériel où se déroulait le culte (de même que celle de la sépulture des défunts). L'époque carolingienne marque une étape importante dans la valorisation du lieu cultuel, comme l'indique le développement d'un rituel spécifique de dédicace de l'église à partir du VIII ${ }^{e}$ siècle. Au $\mathrm{XI}^{\mathrm{e}}$-XII ${ }^{\mathrm{e}}$ siècles, le renversement est devenu complet, puisqu'on attribue alors un caractère absolument nécessaire au bâtiment ecclésial (en même temps qu'un autre renversement doctrinal aboutit à la proclamation pontificale de la Présence réelle du Christ dans les espèces eucharistiques). Le lien entre ces deux questions est fort bien énoncé par Bonizon de Sutri, évêque de Plaisance à la fin du $\mathrm{XI}^{\mathrm{e}}$ siècle : de même que le prêtre qui célèbre la messe transforme le pain et le vin en corps et sang du Christ, l'évêque qui consacre une église transfigure l'édifice matériel en demeure spirituelle de la Trinité et des anges ${ }^{6}$. Plus largement, les clercs grégoriens mettent alors au point une doctrine inédite du lieu sacré, soulignant que si Dieu est partout, il existe cependant des lieux où il est "plus présent » et qui sont donc légitimement voués à son culte. Ils 
affirment surtout qu'il ne peut y avoir de célébration des sacrements sans usage des lieux consacrés. Les édifices cultuels sont alors devenus indispensables au fonctionnement de l'Église, c'est-à-dire à la reproduction de la société dans son ensemble. Le contenant (l'église) est la condition d'existence du contenu (l'Église), le moyen et la forme même de sa réalisation.

Dès lors, il n'est pas déraisonnable de supposer que l'importance acquise par les bâtiments ecclésiaux est étroitement liée à la refondation de l'institution cléricale et à l'accentuation de sa position dominante. Du reste, c'est pour répliquer aux attaques des dissidents (ceux du concile d'Arras, en 1025, ou Pierre de Bruys au siècle suivant), qui sapent les fondements de l'institution en déniant la nécessité des lieux de culte (comme aussi celle des images et des suffrages pour les morts), que les clercs élaborent la nouvelle doctrine du lieu cultuel ${ }^{7}$. Il serait pourtant malencontreux de considérer l'église-bâtiment comme une simple image du pouvoir de l'Église-institution. On gardera plutôt à l'esprit la nécessité d'associer et de mettre en tension les trois significations de la notion d'Ecclesia, qui désigne à la fois la communauté de tous les chrétiens (c'est le sens premier, dérivant du grec eklesia, assemblée), l'édifice (dénommé ainsi parce qu'il accueille la communauté) et l'institution que composent les clercs (sens de plus en plus fréquent à partir du XI ${ }^{e}$ siècle). Ainsi, dans l'édifice, s'entrelacent la symbolisation du pouvoir clérical (puisque ce lieu est celui du recours obligé à la médiation sacerdotale) et celle de la communauté (puisque le bâtiment est l'image de celle-ci, identifiée au corps du Christ, tandis que les fidèles sont les pierres vivantes composant l'édifice-corps de l'église, que la dédicace traite comme une personne à baptiser $^{8}$ ). Si l'on peut affirmer que l'édifice-église réalise idéalement l'Ecclesia, c'est à la condition d'apporter au moins trois précisions : cette Ecclesia est à la fois une et duelle, communautaire et hiérarchique; elle reste prise dans la tension entre ses vicissitudes terrestres (Ecclesia peregrinans) et la perfection de sa réalisation céleste (Ecclesia triumphans); elle met en jeu une assimilation entre l'Église locale (fondée tout particulièrement sur les reliques qu'abrite l'édifice et les figures saintes auxquelles il est identifié) et l'Église universelle (dès lors que, par les sacrements, la communauté locale réalise le corps du Christ et s'identifie à la chrétienté tout entière ${ }^{9}$ ). Enfin, le concept d'Ecclesia intègre le monde social au sein d'un ordre cosmique : il ne désigne pas seulement la communauté des fidèles vivants, mais tout autant celle des défunts et des puissances célestes. Si l'église tend à réaliser l'Ecclesia, c'est donc en tant qu'union ordonnée des vivants et des morts, dans la compagnie des anges et de Dieu.

Cœur matériel et spirituel polarisant l'espace des morts et des vivants, lieu obligé de l'effectuation des rites qui permettent le salut individuel et la reproduction du corps social, concrétion architecturée de l'Ecclesia : on ne saurait trop souligner l'éminence du lieu cultuel, son statut proprement hors du commun. C'est à partir de leur «sursacralisation", sorte de transcription de leur rôle socio-spatial déterminant, qu'il convient donc d'approcher les édifices ecclésiaux. Or, la suréminence architecturale et décorative qui dilate et fait rayonner ces lieux n'est rien d'autre que la manifestation sensible, l'incarnation tangible de ce statut sacral et social. On ne saurait dissocier l'amplification croissante du décor de l'église (par des images bidimensionnelles puis, de plus en plus, en trois dimensions) de ces questions essentielles. 


\section{Emboîtement des seuils et lieu liminaire}

$7 \quad$ Le statut de l'édifice ecclésial (et ses ambivalences) se transcrivent concrètement dans sa constitution et son organisation matérielles. Ce sont elles qu'il convient d'évoquer maintenant.

8 L'édifice ecclésial est certes le lieu positif par excellence, jouant pour cela même un rôle décisif dans la polarisation de l'espace social. Toutefois, plutôt que de considérer ses murs (et ses portes) comme une limite absolue entre une intériorité dite sacrée et un extérieur sans doute trop vite qualifié de profane, il convient de se représenter l'église et son environnement comme un dispositif formé de plusieurs cercles concentriques. En effet, l'église est entourée, non du monde profane, mais d'une enveloppe sacrale, le plus souvent associée au cimetière, c'est-à-dire au statut fondateur de l'ancestralité. Et même lorsque un édifice est dépourvu de fonction funéraire, cette enveloppe est instituée par le rite de dédicace, le plus souvent à environ trente pas autour de l'église ou parfois à une distance qui peut être sensiblement plus grande, comme dans le cas du sacré ban de Cluny $^{10}$. Contrairement à ce que suggère l'expérience actuelle des bâtiments médiévaux, passer la porte d'une église ne signifiait pas (ou seulement par exception) passer du monde séculier au lieu sacré. Que l'on ait affaire à une cathédrale ou à une collégiale entourée des édifices occupant l'enclos canonial, à une abbatiale à laquelle s'adossent les bâtiments voués à la vie monastique ou à une simple église paroissiale ceinte de la terre des morts, la limite du monde ordinaire était repoussée à distance des murs du bâtiment cultuel. On pénétrait dans un espace doté d'un certain degré de sacralité avant même de franchir le seuil de l'église.

9 Les portes de l'église n'en constituent pas moins des seuils importants, d'où l'amplification du décor de celles-ci, surtout à partir de la fin du $\mathrm{X}^{\mathrm{e}}$ et du début du XI $\mathrm{XI}^{\mathrm{e}}$ siècle ${ }^{11}$. Ce n'est toutefois guère le cas des édifices auvergnats traités ici, dont les entrées, à l'exception des portails sud de Mozat et de Notre-Dame du Port, demeurent aniconiques (on notera également que les accès principaux de ces édifices sont, comme il est courant dans les régions méridionales de la France, plus volontiers situées sur les flancs latéraux de la nef qu'à la façade occidentale ${ }^{12}$ ). Quoi qu'il en soit, l'opposition entre l'intérieur et l'extérieur du bâtiment ne saurait être sous-estimée et les murs de l'édifice constituent une enveloppe protectrice particulièrement importante ${ }^{13}$. Ceci apparaît nettement dans le rite de dédicace, où abondent les circulations, alternativement extérieures et intérieures, visant à conforter la valeur de délimitation des murs ${ }^{14}$. Toutefois, cette opposition intérieur/extérieur ne trouve que rarement sa place dans les commentaires que les liturgistes consacrent à l'édifice ecclésial. C'est un peu par exception que Pierre de Roissy, un membre du cercle rigoriste de Pierre le Chantre, à la fin du XII ${ }^{e}$ siècle, met en rapport cette opposition avec la question du décor : «l'église est ornée solennellement à l'intérieur et non à l'extérieur, ce qui indique que toute sa gloire est à l'intérieur $»^{15}$. En fait, cette affirmation, de plus en plus contredite par l'évolution de l'art gothique dont Pierre le Chantre dénonçait le faste croissant, correspond bien mieux aux édifices romans dont il sera question ici. En tout état de cause, sans nullement chercher à minorer l'importance de l'opposition intérieur/extérieur marquée par les murs de l'édifice, il faut souligner qu'il s'agit là d'une « enveloppe » parmi plusieurs autres. Quelle que soit l'importance de la porte de l'église, magnifiée notamment à Saint-Pierre de Mozat où une belle inscription en souligne la valeur, elle n'est qu'un seuil parmi d'autres. 
En effet, si l'unité du lieu sacré est une donnée majeure, elle se combine à une division de plus en plus marquée de ses espaces intérieurs ${ }^{16}$. Après quelques esquisses à Rome, dès le $\mathrm{VII}$ e siècle, les premières séparations isolant le sanctuaire apparaissent à l'époque carolingienne, en relation avec les prescriptions qui interdisent aux laïcs de se trouver à proximité de l'autel au moment de la messe. Une étape ultérieure s'engage à partir du milieu du $\mathrm{XI}^{\mathrm{e}}$ siècle : partout, de la péninsule italienne à l'Angleterre, se généralisent de hauts murs de chancel, afin que les laïcs soient soustraits aux regards des clercs et les clercs à ceux des laïcs (fig. 1) ${ }^{17}$.

Fig. 1

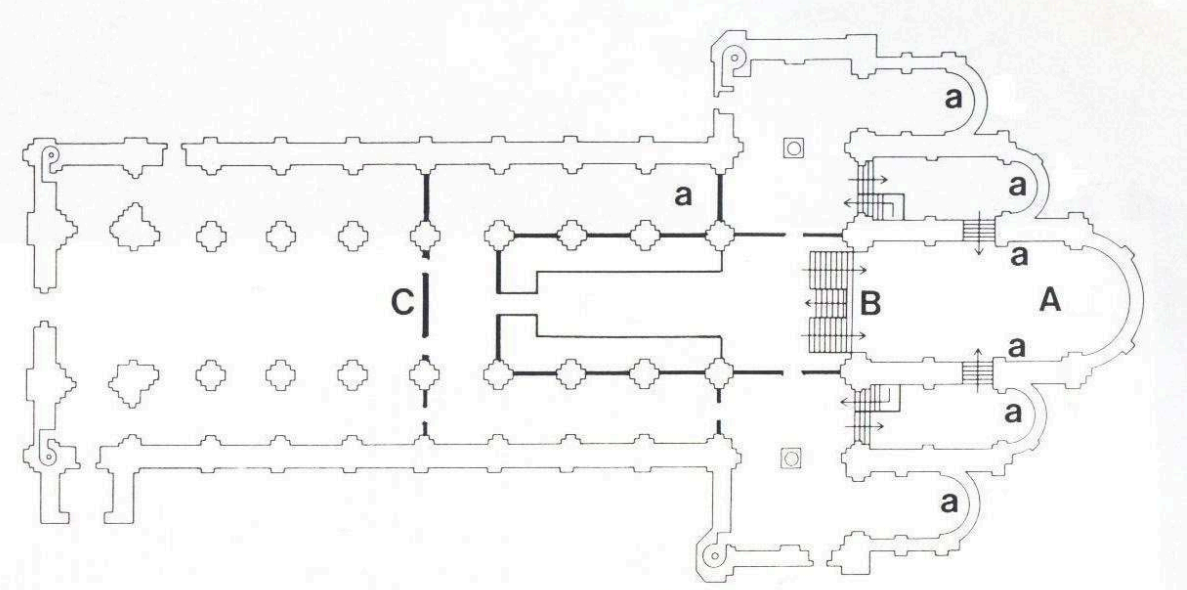

Cathédrale de Cantorbery à la fin du XI e siècle (reconstitution des aménagements liturgiques, d'après A. Klukas). La lettre « $C$ » indique l'autel de la croix, le « B » l'autel majeur, le « $A$ » l'autel matutinal et les « a » les autels secondaires

11 De tels dispositifs, qui excluent physiquement et visuellement les laïcs du lieu de la célébration eucharistique, sont l'expression architecturée d'une distinction sociale majeure, à laquelle la refondation ecclésiale des $\mathrm{XI}^{\mathrm{e}}-\mathrm{XII}^{\mathrm{e}}$ siècles confère une extrême rigueur. Il y a en effet, selon l'expression du Décret de Gratien, "deux sortes de chrétiens ", les uns voués aux affaires du siècle et au mariage, tandis que les autres sont voués, par le célibat et le renoncement aux liens de la parenté charnelle, aux affaires de l'Église. Ils doivent donc, comme le précise Humbert de Silva Candida, être «séparés au sein des sanctuaires par les places et les offices ${ }^{18}$. De fait, lorsque les liturgistes du XII siècle commentent la structure du lieu de culte, ils insistent sur la dualité qui oppose la nef, où se tiennent les laïcs, et le sanctuaire, propre aux clercs ${ }^{19}$. Même si ces deux parties sont associées au sein de l'unité du lieu ecclésial et si la frontière qui les délimite pouvait être franchie par les laïcs en certaines occasions, le chancel (comme les autres éléments séparant les espaces liturgiques) est la matérialisation architecturée d'une distinction sociale majeure. Dès lors, l'expérience physique des lieux devient un vecteur privilégié de l'incorporation de cette dernière. 
Pour autant, cette dualité ne suffit pas à rendre compte des hiérarchies internes de l'édifice et il convient de faire place à une division ternaire, entre la nef (navis, pars anterior), espace des laïcs, le chœur (chorus), où les clercs se réunissent pour les offices, et le sanctuaire (sanctuarium), qui abrite l'autel majeur et dont l'entrée est souvent marquée par la présence de l'arc triomphal ou de quelques marches ${ }^{20}$. De fait, les liturgistes du XIII ${ }^{e}$ siècle glissent vers une telle division tripartite, explicitement énoncée par l'évêque de Mende, Guillaume Durand ${ }^{21}$. Certes, cette tripartition ne concerne pas les édifices les plus modestes, dépourvus de chœur liturgique, et donc bipartites. Mais dès la période qui nous occupe, les bâtiments importants (abbatiales, cathédrales, collégiales), desservis par une communauté de moines ou de chanoines qui se réunissent pour les offices, doivent être considérés comme des édifices tripartites ${ }^{22}$. De fait, c'est à cette structuration tripartite que nous nous réfèrerons dans l'étude des édifices auvergnats et il faudra tenter à chaque fois, dans la mesure du possible, de distinguer la nef des laïcs (avec l'autel de la croix qui lui est propre), le chœur liturgique (avec le chancel qui en marque la limite vers l'ouest), et le sanctuaire (fig. $2)^{23}$.

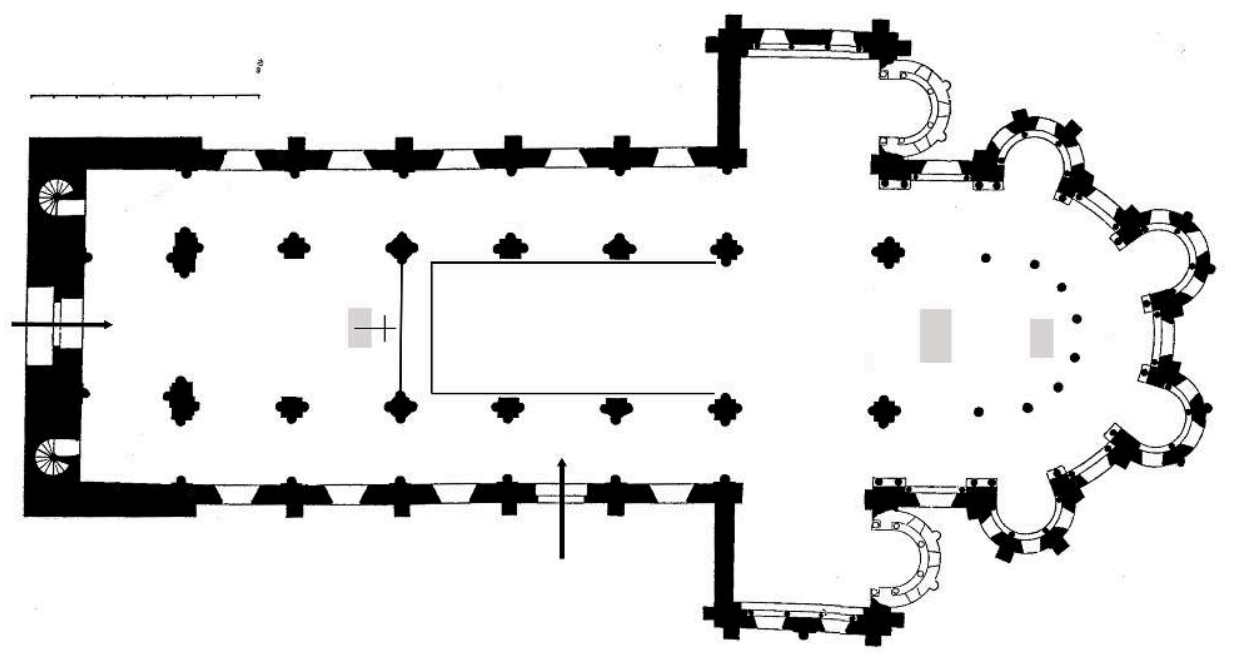

Notre-Dame-du-Port (hypothèse de restitution de l'aménagement liturgique)

Reste à mentionner un espace spécifique sur le statut duquel nous nous interrogerons fréquemment : le déambulatoire, que caractérisent à la fois une grande proximité avec le sanctuaire et une nette différenciation. Les commentaires des liturgistes n'en disent mot et nous sommes fort mal renseignés sur ses usages. Du moins doit-on renoncer à l'interprétation classique du chevet à déambulatoire, comme réponse fonctionnelle visant à canaliser le flux des pèlerins attirés par des reliques prestigieuses (le nom même du déambulatoire, forgé au XIX ${ }^{e}$ siècle, conforte indûment une association avec l'idée de circulation ${ }^{24}$. Tout en tenant compte de la diversité des situations, on doit plutôt admettre que le déambulatoire relève des espaces réservés d'abord aux clercs eux-mêmes et à la célébration des actes liturgiques propres à la communauté cléricale 
en charge du lieu. C'est ce que confirme parfois la présence de grilles à l'entrée du déambulatoire ${ }^{25}$. Et sans doute n'est-ce que de manière très réglée et limitée aux fêtes les plus importantes que le déambulatoire pouvait être rendu accessible aux laïcs qui, par-dessus les grilles entourant le sanctuaire, pouvait alors apercevoir le reliquaire du saint $^{26}$. Si la présence des laïcs dans le déambulatoire est possible, celle-ci ne saurait définir ni la raison d'être ni la nature de ce lieu, dont on peut souligner bien plutôt qu'il vise à entourer et à magnifier le sanctuaire, comme le suggère bien l'usage du terme corona pour désigner cette partie de l'édifice ${ }^{27}$.

Ajoutons que l'unité du lieu sacré et ses divisions internes sont englobées dans un même mouvement, dès lors que le lieu intérieur est traversé par une dynamique axiale que polarisent l'abside et l'autel majeur. C'est là le résultat d'une évolution historique remarquable ${ }^{28}$. En effet, si les grandes basiliques romaines et ravennates témoignaient déjà d'un modèle nettement axialisé, l'époque carolingienne a fait prévaloir une complexité des dispositifs liturgiques, associée à une considérable multiplication des autels. Ils se distribuaient au sein d'un espace ecclésial dont l'architecture matérialisait la bipolarité, avec une abside orientale dédiée à un saint et un massif occidental amplifié, accueillant l'autel du Sauveur (éventuellement ceux de la Vierge dans la crypte et de Michel dans les tribunes). Au cours des $\mathrm{X}^{\mathrm{e}}-\mathrm{XI}^{\mathrm{e}}$ siècles, cette configuration se défait. Et même si certains dispositifs de transition entre intérieur et extérieur suscitent encore une amplification notable de la partie occidentale de l'édifice (narthex, porche, galilée) ${ }^{29}$, on assiste au regroupement de la plupart des autels dans la partie orientale de l'édifice (autel majeur et autel matutinal dans le sanctuaire, auxquels peuvent s'ajouter ceux des absidioles du transept et du déambulatoire; seul l'autel de la croix demeurant fréquemment en avant du chancel, à destination des laïcs $)^{30}$. A mesure que la bipolarité carolingienne s'estompe, l'axialité dynamique du bâtiment, polarisé par l'autel majeur et l'abside, s'affirme comme une caractéristique majeure des édifices occidentaux ${ }^{31}$.

15 Vers 1200, l'évêque Sicard de Crémone exprime cette dynamique axiale de façon remarquable, lorsqu'il associe la longueur de l'édifice ecclésial à la patience "qui supporte les épreuves jusqu'à parvenir dans la patrie céleste $»^{32}$. Cette expression aussi riche que concise suggère d'associer la nef avec l'adversité (le combat spirituel) et l'abside avec la plénitude paradisiaque. Surtout, elle assimile l'axialité longitudinale de l'édifice au cheminement de l'homo viator, au pèlerinage de la vie humaine tendue dans l'espérance du ciel. En quelques mots, Sicard noue habilement la dynamique axiale de l'église et la valeur centrale de la société chrétienne : la quête du salut. L'un et l'autre sont associés à un cheminement (iter), schème omniprésent dans les représentations médiévales de l'espace ${ }^{33}$. Déployant intérieurement l'iter que polarisent l'autel majeur et l'abside, l'édifice cultuel reproduit ainsi, en son intérieur et à une échelle réduite, le modèle du pèlerinage dont il constitue, en tant que lieu sacré, le point d'attraction.

Posons alors que l'édifice ecclésial peut être pensé à la fois comme locus et comme iter. Il est le lieu sacré par excellence, polarisant l'espace social (localement ou plus amplement, selon les flux de pèlerins qu'il draine). Mais son unité sacrale s'ouvre dynamiquement pour déployer, en son sein, un iter scandé par une série de seuils réitérés, à franchir comme autant d'épreuves ( $a d v e r s a$ ) et menant à chaque fois vers une plus grande plénitude du sacré. Paradoxale, cette conjonction entre locus et iter aide à rendre compte de la structure concentrique déjà évoquée, et plus largement de la complexité et des tensions qui font la richesse de ses modes de fonctionnement ${ }^{34}$. On en 
trouve du reste mention dans le rituel de dédicace, dont une formule qualifie l'édifice de «maison de Dieu et porte du ciel» (domus dei et porta celi) $)^{35}$. Cette expression est souvent reprise dans les inscriptions placées à la porte de l'édifice, avec parfois des variantes suggestives ${ }^{36}$. Domus dei indique bien un locus sacralisé par la présence privilégiée du Créateur; mais ce lieu est en même temps conçu comme un seuil, une mise en chemin (porta, via) vers la pleine réunion à Dieu. Telle est l'ambivalence d'un lieu pleinement sacré et néanmoins traversé par la tension d'une quête.

17 C'est à une analyse proche que parvient Didier Méhu, lorsqu'il convoque les notions de locus et de transitus pour rendre compte de l'édifice cultuel ${ }^{37}$. Cette dernière notion met l'accent sur le mouvement vertical qui s'ouvre au sein du lieu sacré, sur la conjonction entre ciel et terre qui en est la justification ultime. Certes, le lieu rituel se donne (et se nomme parfois dans les inscriptions déjà signalées) comme une porte donnant accès au ciel. Mais s'il convient d'intégrer cette dimension verticale à l'analyse du fonctionnement du lieu rituel, il n'en faut pas moins prendre en compte la dynamique interne horizontale qu'exprime la notion d'iter. C'est bien à combiner les trois notions de locus, iter et transitus qu'il convient donc de travailler. Revenons, pour ce faire, à notre modèle des cercles concentriques et des seuils emboîtés. Certes, les inscriptions associées à la porte de l'édifice suggèrent une assimilation entre celle-ci et la porte du ciel, ce qui tendrait à projeter l'ensemble de l'espace intérieur dans la dynamique ascendante du transitus. Pourtant, une fois le seuil passé, l'immédiateté du contact avec le ciel est démentie par un nouvel appel à la patience, au cheminement. L'accès au ciel se distend en un nouvel iter, qui est aussi la condition du maintien des hiérarchies terrestres et de leur matérialisation pratique dans l'édifice cultuel. De nouveaux seuils jalonnent cet iter, au chancel d'abord, puis au niveau de l'arc triomphal. Finalement, c'est essentiellement dans le sanctuaire lui-même, autour de l'autel majeur, que s'opère, dans le temps de la célébration eucharistique, la jonction entre terre et ciel. Du reste, l'un des traits les plus constants du décor du sanctuaire est la présence, dans la conque absidiale ou à la voûte de la travée droite, d'une figuration peinte de la Majesté divine (et bien que nous n'en ayons pas conservé de traces en Auvergne, il paraît raisonnable de supposer que les absides des églises étudiées ici étaient ornées d'un décor de ce type) ${ }^{38}$. A Saint-Nicolas de Tavant, Dieu en majesté est même entouré d'anges qui tendent vers lui hostie et calice, de sorte que l'image constitue la matérialisation visible de ce que la liturgie accomplit normalement de manière invisible, à savoir le transport des offrandes eucharistiques jusque sur l'autel céleste ${ }^{39}$. Qu'elle soit ou non accompagnée de figurations aussi explicites, la théophanie des décors absidiaux rend sensible la conjonction, réalisée lors de la célébration de la messe, entre la liturgie terrestre et la liturgie céleste, dans la pleine présence de Dieu et des anges ${ }^{40}$. Ainsi, le sanctuaire (avec l'autel majeur) est le cadre d'un ultime paradoxe. $\mathrm{Au}$ cœur des cercles concentriques évoqués plus haut, il est le lieu le plus intérieur de tous. Et pourtant, voici qu'il s'ouvre à son tour, échappant à ses propres limites. L'iter ecclésial, parvenu à son terme, bascule cette fois pleinement dans la verticale du transitus, pour opérer une conjonction de la terre et du ciel.

18 En résumé, si le transitus est la justification ultime du lieu sacré, celui-ci ne se laisse pas absorber entièrement par une telle verticalité - sinon idéalement. En pratique, s'ouvre au sein du locus la dynamique horizontale d'un iter dont la prise en compte est indispensable, dès lors qu'on souhaite rendre compte de l'organisation interne du lieu et de celle de son décor. Une fois encore, on peut souligner le caractère paradoxal de l'édifice ecclésial, que l'on qualifiera de lieu liminaire. De fait, l'inscription qui orne le 
porche nord de l'abbatiale Saint-Pierre de Mozat désigne l'église, dans laquelle elle invite à pénétrer, comme «templum » et comme " limina Christi » (voir chapitre II). Lieu liminaire, parce que le lieu rituel n'a la plénitude d'un locus sacré que dans la fonction de polarisation qu'il exerce vis-à-vis de l'extérieur. Lieu liminaire surtout, parce que, en son intérieur, s'ouvre en une série de seuils jalonnant un iter horizontal et parce que, celuici bascule ultimement dans la verticalité d'un passage (transitus) entre terre et ciel. Locus sacré traversé par la dynamique de l'iter, l'édifice ecclésial est un paradoxal lieu liminaire qui ouvre, dans le pèlerinage terrestre des hommes, le seuil d'une conjonction verticale avec le monde divin. Si l'on admet cette hypothèse, les phénomènes de liminarité ne devraient pas être associés de façon étroite aux seules portes ou portails mais pourraient être tenus pour une caractéristique généralisée du lieu ecclésial dans son ensemble.

\section{Les chapiteaux, points de concentration de forces}

Dans les chapitres qui suivent, nos analyses vont se concentrer sur les chapiteaux, ou plus exactement sur les relations entre les chapiteaux et le lieu rituel qu'ils contribuent à magnifier. On ne peut cependant ignorer la nécessité d'une saisie aussi globale que possible de l'édifice ecclésial comme lieu d'images, ce qui suppose de considérer la totalité de son décor : peinture murale, croix peintes ou sculptées, reliquaires et décor funéraire éventuel, ornements du chancel et des autels, objets liturgiques multiples, etc. Certes, hormis des reliquaires remarquables, les édifices auvergnats ont été presque entièrement vidés de leur mobilier liturgique d'origine et, sauf exception, ont perdu l'essentiel de leur décor peint médiéval. Pourtant, même lorsqu'ils ont entièrement disparus, ces éléments doivent être pris en compte dans l'analyse : si leurs caractéristiques demeurent hypothétiques, leur présence à peu près certaine ne peut être ignorée, dès lors qu'elle contribuait à la constitution du lieu rituel comme lieu d'images. Ainsi, nous savons avec un degré de certitude raisonnable que les murs et les colonnes des églises étudiées ici étaient recouverts d'enduits peints. Seule Saint-Julien de Brioude en a conservé des fragments bien visibles, qui donnent une idée du chatoiement chromatique et de la puissance des motifs que le décor peint pouvait faire jouer au sein de l'édifice. 
Fig. 3

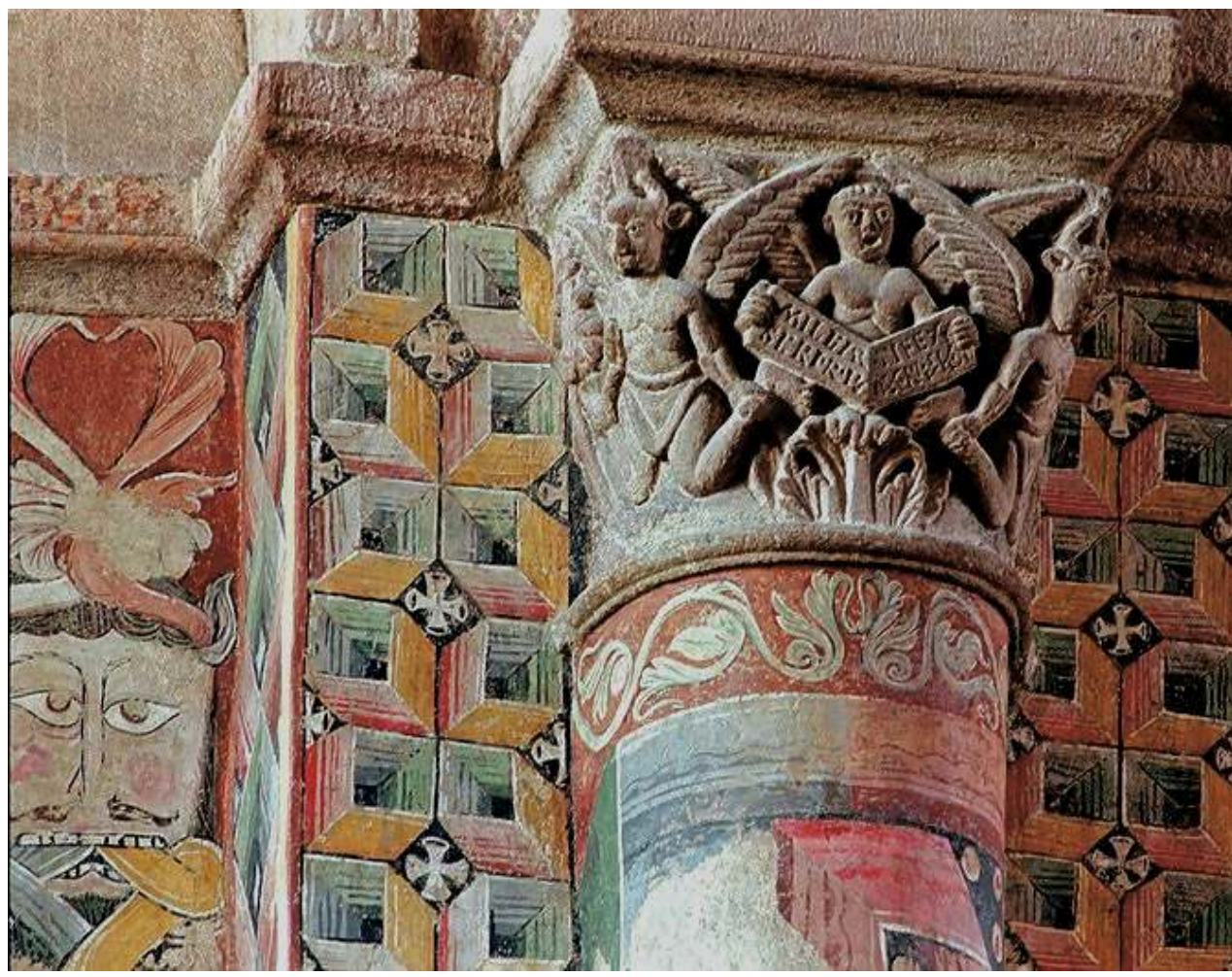

Saint-Julien de Brioude : chapiteau sculpté et décor peint

20 Toutefois, les relevés attentifs de Dominique Allios ont montré l'omniprésence d'une polychromie d'époque romane sur toutes les surfaces intérieures de l'église de SaintNectaire ${ }^{41}$. Les chapiteaux étant eux-mêmes rehaussés de polychromie, il convient de les appréhender en imaginant un jeu de continuités et d'intensifications par rapport au décor coloré des murs et des colonnes elles-mêmes.

Rien ne permet, s'agissant des édifices pris en compte ici, de restituer un décor peint porteur d'une iconographie plus élaborée. Toutefois, comme on l'a dit, il est peu probable que la conque absidiale soit demeurée vide de tout décor : une figuration théophanique y est hautement vraisemblable. A défaut, des motifs évoquant une dimension céleste (des étoiles, par exemple) pourraient y avoir trouvé place. A l'autre extrême du spectre décoratif, il faut faire mention des faux appareils peints, qui ornent de manière récurrente les murs intérieurs des églises médiévales (on en a conservé d'infimes traces, d'époque romane, à Saint-Nectaire ${ }^{42}$ ). C'est là un usage qui déconcerte d'autant plus nos manières de voir contemporaines que ces appareillages peints, loin d'être des cache-misère, recouvrent souvent des blocs soigneusement taillées ${ }^{43}$. Il faut donc attribuer à la représentation peinte des pierres une valeur supérieure à celle de la pierre taillée apparente, comme si l'appareil peint éloignait de la stricte matérialité de la pierre, maintenue pour l'essentiel à l'extérieur, et opérait une transposition vers un registre plus spirituel, plus apte à exprimer les valeurs associées à l'intériorité du lieu sacré et à l'image des fidèles comme "pierres vivantes" de l'Église. Habillant les pierres d'une parure continue, le décor intérieur peint pourrait être considéré comme le vecteur d'une spiritualisation de l'édifice matériel, d'un glissement vers son statut de lieu spirituel, donnant corps à la communauté chrétienne. 
Éléments constitutifs de la globalité du lieu d'images, les chapiteaux n'en constituent pas moins des supports spécifiques. Même s'ils partagent certaines caractéristiques avec l'ensemble du décor sculpté d'une église (tridimensionnalité, mode de présence intensifié de la corporéité) ${ }^{44}$, ils requièrent un type d'analyse adapté, qui ne saurait pas davantage être une simple transposition de principes élaborés à propos des peintures murales ou d'autres supports encore. C'est à la construction de telles analyses que le présent travail entend contribuer. Que les chapiteaux constituent des images-lieux singulières, leur histoire le souligne assez. Il est peu de supports d'images qui soient aussi spécifiquement associés à une époque bien précise du Moyen Age. Même s'ils n'avaient pas disparu durant le haut Moyen Age ${ }^{45}$, c'est à partir de la fin du $\mathrm{X}^{\mathrm{e}}$ et du début du $\mathrm{XI}^{\mathrm{e}}$ siècle que l'on peut situer un nouvel essor de la production de chapiteaux sculptés à décor végétal, géométrique, à figures, puis, de plus en plus fréquemment, historiés ${ }^{46}$. Jusqu'à la fin du XII ${ }^{\mathrm{e}}$ ou au début du XIII ${ }^{\mathrm{e}}$ siècle, les chapiteaux sont l'un des lieux privilégiés où s'exprime l'inventivité volontiers débridée des artistes romans. Et, si elle est parfois associée à l'idée d'un "retour à l'antique ", cette production n'en présente pas moins un écart structurel majeur par rapport aux usages du monde romain : tandis que les chapiteaux de l'Antiquité investissaient l'édifice d'un principe de régularité et d'une harmonie fondée sur la scansion de formes tendanciellement identiques, ceux de l'époque romane se caractérisent au contraire par la virtuosité avec laquelle, du moins sur les chantiers les plus importants, est mis en œuvre un principe de diversification (varietas) ${ }^{47}$. Assumer cette logique de variation maximale, à l'opposé des principes de l'architecture classique, était la condition de la profusion inventive dont témoignent les chapiteaux de l'époque romane. Mais leur succès fut aussi intense que bref. Avec l'art gothique, les chapiteaux cessent d'être des supports d'images aussi privilégiés et diversifiés; ils tendent à être reconduits vers un principe de plus grande similarité et vers une végétalité dominante, sinon exclusive ${ }^{48}$.

Tentons maintenant de mieux saisir le statut spécifique des chapiteaux et leur mode de fonctionnement au sein de l'édifice ecclésial, en les considérant tout d'abord dans leur rapport aux colonnes qui les soutiennent, puis en relation avec les arcades et les murs qu'ils contribuent à soutenir, et enfin en les inscrivant dans la totalité de l'édifice.

Le chapiteau ne peut être dissocié de la colonne dont il est le sommet et à laquelle s'attache une forte valeur symbolique. Les nombreux commentaires exégétiques relatifs aux deux colonnes de l'entrée du Temple de Salomon (I Rois 7, 13-22) ont constitué un abondant réservoir de références susceptibles d'entretenir la valorisation des colonnes de l'édifice chrétien. Mais rien ne l'indique mieux que l'insistance des descriptions d'églises sur l'abondance et la beauté des colonnes, dont on donne volontiers le nombre précis (70 pour la cathédrale de Clermont au $\mathrm{V}^{\mathrm{e}}$ siècle, selon Grégoire de Tours, 193 à Saint-Denis en 799 ${ }^{49}$. On sait aussi l'importance que Suger attribuait aux deux rangées de douze colonnes dont il dota le nouveau chevet de son abbatiale, ainsi que le symbolisme qu'il leur attribuait (les apôtres et les prophètes).

Une autre expression de la valorisation des colonnes et des chapiteaux est la pratique consistant à y placer des reliques. Quoique tout à fait passionnant, un cas comme celui du cloitre de Moissac, où le chapiteau du martyre de Pierre et Paul est creusé d'une cavité dans laquelle étaient placées des reliques, n'est cependant pas le plus pertinent pour notre propos, dans la mesure où la présence des reliques y est directement liée au sujet représenté et valorise un emplacement jouant un rôle spécifique dans la liturgie monastique ${ }^{50}$. Importent davantage ici des témoignages comme celui de la Chronique de 
Thietmar de Merseburg, qui rapporte que, lors de la construction de la cathédrale de Magdeburg, l'empereur Otton III ordonna de placer des reliques « au sommet de toutes les colonnes $»^{51}$. On ne sait trop s'il faut comprendre que les reliques étaient logées entre la colonne et le chapiteau ou à l'intérieur de celui-ci. En tout cas, une pratique similaire est attestée pour de nombreux autres édifices, notamment à Saint-Michel de Hildesheim ou encore au Mont-Cassin, où il est précisé que les reliques furent enfermées dans de petites pyxides de bronze avant d'être placées dans les chapiteaux ${ }^{52}$.

Un exemple plus tardif, mais particulièrement suggestif, concerne l'abbaye de Lagrasse. Mise par écrit au milieu du XIII e siècle par un moine du lieu, les Gesta Karoli Magni évoquent la construction de l'abbatiale à l'époque de Charlemagne et indiquent : « que tous les chapiteaux soient creux, car nous y mettrons assez de reliques afin que ce lieu soit gardé de toute tempête et de toute foudre par la volonté de Dieu ${ }^{53}$. Il ne s'agit pas seulement d'une mention littéraire, car l'étude attentive de nombreux chapiteaux a permis de relever la présence, d'une part, de petites cavités sur les faces latérales (qui pouvaient soit donner lieu à des incrustations de pierres colorées ou de verroterie, soit contenir une relique masquée par un décor de stuc) et, d'autre part, de cavités plus importantes sur la face supérieure du chapiteau, vraisemblablement destinées à accueillir des reliques ${ }^{54}$. Certes, les chapiteaux ne sont pas les seuls emplacements de l'édifice où l'on place des reliques. Outre les autels, qui en sont nécessairement pourvus, il est fait mention, au Mont-Cassin, de reliques placées dans l'arc triomphal et à la voûte de la chapelle Saint-Jean et, à Lagrasse même, dans l'arc de la croisée du transept, ainsi qu'au-dessus des fenêtres éclairant l'abside ${ }^{55}$. D'après ces témoignages, les chapiteaux sont donc investis d'une fonction apotropaïque, qu'ils doivent certes aux reliques dont ils sont truffés et qu'ils partagent avec d'autres parties de l'édifice. Du moins peut-on en déduire qu'ils constituent des emplacements fortement valorisés et assez importants pour assumer, de manière privilégiée, une telle fonction.

Le statut des chapiteaux appelle d'autres remarques encore. Notons d'abord que le latin utilise, pour les désigner, le terme «capitellum $»^{56}$. De manière attendue, Isidore de Séville explique que les capitella se nomment ainsi parce qu'ils constituent les têtes des colonnes (" capita columnarum »; Etimologiae, XV, 8, 13). Cette association avec la partie supérieure du corps, tenue pour la plus spirituelle, est un élément important de valorisation des chapiteaux qui sont, par conséquent, à la colonne ce que le chevet est au reste de l'église (caput ecclesiae). À partir de Bède et Raban Maur, l'exégèse propose une lecture allégorique dans laquelle les colonnes représentent les apôtres et les docteurs évangéliques, tandis que leur partie supérieure renvoie aux pensées (cogitationes) de ces derniers ${ }^{57}$. Mais ces commentaires concernent les deux colonnes placées à l'entrée du Temple de Salomon et il est probable que l'expression «capita columnarum » se réfère au sommet des colonnes plutôt qu'à leurs chapiteaux. Puis, dans les commentaires liturgiques du XII ${ }^{e}$ siècle, c'est à l'édifice ecclésial que s'appliquent les indications initialement associées au Temple. Elles sont alors actualisées et amplifiées : ainsi, à partir d'Honorius Augustodunensis, puis chez Sicard de Crémone, les colonnes sont assimilées non plus aux apôtres mais aux évêques, ce qui souligne le phénomène de cléricalisation de l'Église. Le sommet des colonnes est alors associé à l'esprit des évêques ("mentes episcoporum »), tandis que Sicard de Crémone ajoute à cette mention une claire référence aux chapiteaux eux-mêmes, associés aux paroles de l'Écriture. Ce type d'exégèse invite donc à percevoir le chapiteau comme la partie pensante, ou parlante, de la colonne. Certes, ces commentaires ne nous dévoilent pas ce que sont les chapiteaux (ou n'importe quelle autre partie de l'édifice). Ils ne constituent qu'un 
discours parmi d'autres possibles. Mais il n'est pas indifférent que le chapiteau y soit considéré comme l'expression (pensante ou parlante) du corps-colonne : c'est un objet visible, mais aussi, d'une certaine manière, une voix. Et une voix à la fois mélodique et prolixe, lorsque se multiplient motifs végétaux, figures et scènes historiées. En tout cas, à côté de la présence physique fréquente de reliques, ces commentaires, qui optent pour des référents allégoriques éminemment spirituels, confirment la très forte valorisation qui s'attache aux chapiteaux ${ }^{58}$.

Bien que l'exégèse liturgique se concentre sur cet aspect, on ne saurait considérer les chapiteaux uniquement dans leur rapport aux colonnes qu'ils couronnent. Et, si ces mêmes textes insistent sur la fonction de soutien des colonnes ${ }^{59}$, ils ne mentionnent pas cette fonction à propos des chapiteaux, alors que ces derniers sont précisément les points où s'opère la jonction des colonnes avec les arcs et les murs qu'elles soutiennent. De fait, l'idée d'une position intermédiaire des chapiteaux a parfois été mise en relief dans les études sur l'art médiéval : à une transition architecturale (entre la circularité de la colonne et la section carrée de la retombée de l'arc supporté; entre la verticalité de la colonne et la courbure de l'arc; entre la linéarité de la colonne et la planéité des murs), viendrait s'associer une position intermédiaire (ou charnière) entre le monde terrestre et le domaine céleste, entre l'homme et la divinité ${ }^{60}$. On peut ajouter que les chapiteaux doivent aussi être perçus comme éléments de l'arcade dans laquelle ils s'insèrent et que cette arcade vaut comme passage (voire comme " porte du ciel»), tout particulièrement s'il s'agit de l'arc triomphal ou même des arcs doubleaux qui scandent la nef ${ }^{61}$.

Faut-il véritablement attribuer aux chapiteaux ce statut de jonction ou de passage? On pourrait alors les considérer comme des supports liminaires (accentuant le caractère de liminarité que l'on a attribué à l'édifice ecclésial dans son ensemble). En tout cas, on peut tenir les chapiteaux pour des points d'image hyperdensifiés, des points de concentration de forces, grouillants d'énergie et de vie. Et on peut aisément comprendre que cet art des articulations tensives qu'est l'art roman exploite au maximum les potentialités d'un tel support (tandis que l'art gothique, davantage porté au lissage unificateur, tend à s'en détourner). Plus précisément, il faut restituer un probable contraste, au sein de l'édifice, entre un décor sculpté concentré sur des emplacements qui intensifient les valeurs de liminalité (portail, modillons, chapiteaux, etc.) et un décor peint, lié aux surfaces murales et aux voûtes.

Il convient enfin d'évoquer l'effet associé à la présence des chapiteaux au sein de l'édifice tout entier. Le plus remarquable semble ici la manière dont les chapiteaux (avec les colonnes et d'autres éléments encore) scandent la nef, tout à la fois de leur répétitivité structurelle et de leurs variations formelles internes. En rythmant la progression longitudinale de la nef et des bas-côtés, ces points de force que sont les chapiteaux contribuent (que leur décor soit majoritairement végétal ou plus historié) à musicaliser l'iter qui traverse le lieu rituel. Tandis que cette remarque vaut pour les chapiteaux de la nef, elle ne saurait s'appliquer à ceux du sanctuaire, pour lesquels prévaut une logique tout à fait différente. Au contraste, particulièrement fréquent dans les églises d'Auvergne, entre les demi-colonnes engagées de la nef et les colonnes pleines du sanctuaire, s'ajoute l'opposition évidente entre la disposition rectiligne des premières et l'agencement semi-circulaire des secondes. L'effet produit est particulièrement remarquable : le demi-cercle des colonnes du rond-point valorise le lieu qu'elles encadrent; il solennise la sacralité du sanctuaire et en renforce la capacité 
de polarisation. En même temps, ce dispositif pointe avec vigueur vers la conque absidiale, porteuse d'un décor sans doute théophanique ou du moins céleste. En bref, la disposition différenciée des colonnes et des chapiteaux dans la nef et dans le sanctuaire contribue à articuler iter, locus et transitus : l'iter longitudinal prévaut dans la nef, tandis que le sanctuaire, locus sacré par excellence polarisant l'iter de la nef, est aussi le lieu privilégié du transitus vertical qui met en relation liturgie terrestre et liturgie céleste.

Une remarque plus générale s'impose encore. L'une des caractéristiques les plus marquantes des chapiteaux est leur omniprésence dans l'édifice. Ils prennent littéralement possession de toutes ses parties, tant horizontalement (depuis le narthex, la nef et les bas-côtés jusqu'au sanctuaire et au déambulatoire) que verticalement (avec leur déploiement à l'étage du narthex, sur les arcs des tribunes, les fenêtres principales et les baies hautes du vaisseau central ou du sanctuaire). La même chose vaut, en mineur, pour l'extérieur de l'église, notamment aux fenêtres et aux arcatures. Les chapiteaux investissent donc la totalité du lieu ecclésial, et pourtant, comme on l'a dit, ce sont des points d'image extrêmement concentrés. De la jonction de ces deux aspects - nature très localisée de chaque chapiteau et expansivité de leur distribution - résulte une certaine tension. Et c'est précisément pour rendre compte d'une telle conjonction entre discontinuité et dissémination qu'il est nécessaire d'élaborer un mode d'analyse spécifique, bien distinct par exemple de celui que peut susciter un cycle peint ou un portail. On considérera donc les chapiteaux comme des points d'images saturés d'énergie surconcentrée, mais prenant néanmoins possession de la totalité du lieu ecclésial. C'est à analyser l'agencement de ces séquences discontinues de points de force, ainsi que les types de rapports qui peuvent se nouer en leur sein, que le présent travail sera consacré.

Au total, les remarques avancées ici convergent pour souligner l'extrême valorisation des chapiteaux à l'époque romane, ce que l'amplification débridée de leur décor suffirait du reste à confirmer. Elle n'en est pas moins confirmée tant par la pratique qui fait des chapiteaux des réceptacles privilégiés de reliques contribuant à la protection de l'édifice que par les commentaires des liturgistes qui associent les chapiteaux à la tête, à la pensée ou à la parole, bref à la partie la plus éminente et la plus spirituelle de la personne. Enfin, on a pu souligner la double valeur de liminarité et de concentration énergétique qui s'attache au chapiteau : il semble ainsi intensifier un trait caractéristique de l'édifice ecclésial tout entier et paraît correspondre particulièrement bien au caractère tensif de l'art roman. Mais rien n'exprime mieux l'importance attribuée aux chapiteaux (ou au binôme colonne-chapiteau) que les scènes de donation que l'on analysera en détail dans les chapitres III et IV. Jouant d'une classique synecdoque, le chapiteau (seul ou avec sa colonne) qu'offre un Ranulfus, à SaintNectaire, ou un Stephanus, à Notre-Dame du Port, concentre en lui assez de force pour exprimer la valeur attachée à l'édifice ecclésial tout entier.

\section{Distribution des chapiteaux et méthode cartographique}

Il s'agit donc d'analyser des réseaux (lignes et nœuds) constitués à partir de points d'images à la fois discontinus et déployés dans la totalité du lieu rituel, en récusant d'emblée deux approches particulièrement fréquentes. L'une d'elle affirme que les chapiteaux, avec leurs différents types de décor, végétal, à figures ou historié, sont 
disposés de façon aléatoire au sein de l'édifice, sans logique spatiale particulière : seules des contraintes techniques, liées notamment à l'avancement du chantier, détermineraient l'emplacement attribué à chaque chapiteau, de sorte qu'il serait vain de rechercher, entre eux, des relations spécifiques, et plus encore de se soucier d'une quelconque logique mettant en rapport décor sculpté et organisation interne du lieu rituel. A l'inverse, l'autre posture recourt à la notion de programme, classique dans le champ de l'histoire de l'art médiéval et renvoyant plus ou moins directement aux conceptions d'Émile Mâle, qui faisait de l'art le véhicule d'un enseignement formulé par l'Église ${ }^{62}$. Elle incite à attribuer à un ensemble d'images une cohérence thématique stricte, assimilable à celle d'un discours théologique ou pastoral.

Même si certains auteurs se sont efforcés d'en proposer un usage rénové et assoupli, la notion de programme présente de sérieux inconvénients ${ }^{63}$. Difficilement dissociable de ses usages politiques, informatiques ou génétiques, ce terme, appliqué à l'histoire de l'art, incite presque immanquablement à postuler une intentionnalité séparée de l'exécution de l'œuvre et préexistant au processus de sa production. Il est dès lors difficile d'échapper à la vision conventionnelle qui distingue le concepteur de l'œuvre (un clerc) et l'artiste qui la réalise, pour mieux ramener la signification de l'œuvre aux énoncés doctrinaux que le premier transmet au second afin qu'il les «mette en forme ». Or, s'il est évident que les clercs commanditaires des œuvres ont pu intervenir dans leur conception, ils n'ont jamais exercé qu'un contrôle relatif sur la production de celles-ci. Plus important encore (et sans même reprendre la critique du logocentrisme des approches iconographiques traditionnelles), la notion de pensée figurative autorise un tout autre type d'analyse : si le processus de production de l'œuvre est un véritable acte de pensée, alors une part considérable de la signification des images tient moins à la formulation des énoncés préalables qui peuvent à l'occasion en constituer le point de départ qu'à ce qui se joue dans le déploiement du travail plastique lui-même. Enfin, la notion de programme s'entend presque toujours comme "programme iconographique ", de sorte qu'elle conduit à dissocier les représentations classiquement qualifiées d'iconographiques et les éléments ornementaux, ce qui revient en pratique à exclure ces derniers du champ de l'analyse ${ }^{64}$.

Difficile à dissocier des pièges mentionnés à l'instant, la notion de programme semble particulièrement inadaptée au cas des chapiteaux qui, du fait de la discontinuité et de la forte dispersion qui les caractérisent, ne sauraient présenter le même mode de cohérence ou faire jouer les mêmes types de relations qu'au sein d'un portail, beaucoup plus ramassé et fortement noué sur lui-même, ou dans un cycle peint, inscrit dans une continuité au moins apparente ${ }^{65}$. Finalement, les deux postures critiquées ici s'épaulent mutuellement. A rechercher un degré de cohérence et d'unité qui ne saurait correspondre au mode de fonctionnement propre de ces réseaux discontinus que forment les chapiteaux, les partisans d'une logique programmatique de facto introuvable font le jeu des tenants d'une disposition aléatoire et d'une absence complète d'ordre - laquelle n'est généralement affirmée qu'au regard d'une notion d'ordre et de cohérence trop rigide pour être pertinente.

C'est à explorer une voie échappant à ces deux écueils inverses et adaptée à la spécificité des ensembles de chapiteaux que l'on s'emploiera ici : ni pur désordre, ni raide cohérence programmatique. Il s'agira, pour cela, de rendre compte de ce que l'on dénomme l'agencement des chapiteaux, soit une notion plus englobante et plus souple que celle de programme. Saisir comment ces réseaux discontinus d'images se déploient 
au sein du lieu rituel suppose de mettre en évidence des types de rapports particuliers et éminemment variables : des effets de cohérence locale, des correspondances souples et des oppositions, proches ou à distance, des échos et des ponctuations, des chaînages et des séquences dynamiques, des séries transformationnelles, sans négliger de possibles flottements qu'on évitera toutefois de qualifier trop vite d'« incohérences » (car le fait qu'il existe des zones à plus forte densité relationnelle et d'autres plus « lâches » est en lui-même significatif). Le caractère discontinu et fortement disséminé du réseau d'images constitué par les chapiteaux invite à concevoir celui-ci comme un tissu associatif qui n'est pas d'une seule pièce, susceptible qu'il est de combiner des nœuds fermes et des associations plus souples au sein d'un tramage dont les fils peuvent jouer par endroits de manière assez distendue.

On se demandera encore comment le réseau des images-chapiteaux interfère avec la structure du lieu ecclésial. Là encore, on peut d'emblée récuser tant l'idée d'une indifférence totale des chapiteaux vis-à-vis de la structure du lieu rituel que le postulat de leur complète adéquation à celle-ci. Il s'agit plutôt de cerner comment un réseau d'images est traversé et partiellement informé par la structure du lieu rituel, mais aussi comment, en retour, il contribue à activer et à rendre sensible celle-ci (voire peut-être à y constituer des lieux ou des champs ayant une charge symbolique qui le reconfigure et en fait jouer la structure). Il s'agira tout particulièrement de préciser dans quelle mesure la distribution des chapiteaux est affectée par (et joue de) l'iter longitudinal qui traverse l'édifice ecclésial. D'éventuelles coïncidences entre les particularités du décor sculpté et les divisions liturgiques du lieu rituel devront être soumises à vérification. Mais il est clair qu'elles ne sauraient être conçues comme une adéquation mécanique des unes aux autres, mais plutôt comme des modes de différenciation tendanciels. C'est pourquoi il s'agira moins, dans notre analyse, de découper d'éventuelles zones (parties de l'édifice qu'on supposerait dotées d'un décor homogène et spécifique) que des ensembles aux contours flous et seulement partiellement différenciés. Ainsi, on posera, à titre d'hypothèse, que tout en faisant écho aux découpages liturgiques internes du lieu rituel, la distribution des chapiteaux peut également (se) jouer de ces délimitations, notamment pour créer des points d'embrayage et assurer des passages d'une partie à l'autre de l'édifice ${ }^{66}$. En tout état de cause, rendre compte de l'agencement des chapiteaux au sein du lieu rituel suppose de mettre en évidence des types de relations spécifiques et multiples, tant entre les chapiteaux eux-mêmes qu'entre le réseau d'images qu'ils forment et la structure d'ensemble du lieu rituel.

C'est à cette fin que l'on a mis en œuvre une méthode cartographique, permettant une formalisation synthétique de la totalité du décor sculpté (intérieur) d'un édifice. Atteindre une telle vision d'ensemble impose de ramener chaque chapiteau à un codage élémentaire (en l'occurrence par lettres et couleurs), susceptible d'être porté sur le plan de l'édifice. Cela suppose le passage à un degré d'abstraction et de généralisation qui pourrait aisément être dénoncé comme une perte d'information et comme une démarche excessivement réductrice. Il convient plutôt d'être conscient de ce péril et de tenter de le maîtriser. On a choisi d'assumer résolument cette option, qui a l'avantage de décoller l'analyse des lectures iconographiques conventionnelles, trop soucieuses peut-être de la littéralité particulière de chaque scène, pour orienter vers des niveaux de sens plus génériques, dont on a de multiples raisons de penser qu'ils jouaient un rôle majeur dans le fonctionnement des images médiévales et en particulier dans les modes d'association et d'interaction établies entre elles ${ }^{67}$. 

de chaque chapiteau est la condition d'une cartographie d'ensemble, indispensable pour faire apparaitre les relations repérables entre tous les chapiteaux de l'édifice. Or, ce n'est qu'après s'être efforcé d'établir la totalité de ces relations qu'il est possible de raisonner sur leur fonctionnement global et donc sur la distribution d'ensemble des chapiteaux au sein de l'édifice ecclésial. Par le recours à l'abstraction et à la visualisation synthétique, la méthode cartographique permet ainsi d'analyser, de manière systématique, l'agencement des chapiteaux au sein de chaque édifice (c'est donc aussi un outil qui permet une étude sérielle, en facilitant la comparaison entre plusieurs édifices, dans le but de repérer d'éventuelles constantes, ainsi que les transformations qui peuvent s'opérer de l'un à l'autre). En dépit d'essais forts intéressants ${ }^{68}$, il nous semble qu'un tel travail n'avait jamais été mené avec tout le degré de systématicité requis. Ce qui revient à dire que l'on discourt bien souvent sur la cohérence ou l'incohérence de la disposition des chapiteaux, sans s'être vraiment donné les moyens d'analyser complètement les types de rapports établis entre eux, et moins encore la distribution spatiale de ces différents types de rapports. imagination. Elle a été mise au point au cours d'un travail de plusieurs années, en un processus d'allers et retours entre nos tentatives de formalisation et la complexité des analyses que chaque édifice appelait. Il s'agissait, au fil des modifications apportées à nos cartographies successives, de rechercher la solution qui, malgré les contraintes ou les biais dérivant des choix opérés, serait à la fois la plus cohérente et la plus efficace pour mettre en évidence les phénomènes remarquables qui apparaissaient. Il va de soi que l'état actuel de notre méthode n'est pas sans défaut et pourrait certainement être encore amélioré.

41 Il n'en reste pas moins (et c'est, pourquoi l'occulter ?, tout le plaisir qui s'est attaché à cette enquête) que l'exercice de cartographie de chaque édifice nous a permis de découvrir, à chaque fois et pour notre plus grande surprise, des réseaux de relations et des agencements dotés de propriétés remarquables dont, malgré la fréquentation des édifices eux-mêmes, nous ne soupçonnions pas l'existence. Il peut paraître paradoxal de mettre en évidence, par la cartographie, des relations et des propriétés distributives qui ne semblent pas perceptibles dans l'édifice lui-même. Certains en concluront peutêtre que ce constat condamne notre démarche à l'inutilité. Nous pensons au contraire que l'analyse des œuvres médiévales ne doit être bornée ni par l'intentionnalité attribuée aux concepteurs, ni par la conscience perceptive des "récepteurs ». Elle doit porter avant tout sur l'image-lieu en tant qu'elle existe objectivement comme dispositif situationnel à la fois sémantique, ornemental et efficace (et c'est seulement une fois cette dimension prise en compte dans toutes ses implications, qu'il peut être loisible de s'interroger sur les modalités diversifiées de réception et d'usage).

S'agissant des chapiteaux eux-mêmes, on peut penser que la tension entre une impression initiale d'absence de cohérence, liée à un réseau discontinu et fortement disséminé de points d'images, et l'existence de relations, voire d'effets de structure relativement cachés (ou du moins susceptibles de se donner lentement, dans le temps réitératif d'une longue familiarité) fait partie du mode de fonctionnement mis en jeu par ces images-lieux bien spécifiques. Les thématiques privilégiées par les chapiteaux ne se laissent pas toujours identifier aisément, ni même répartir entre les catégories du Bien et du Mal : plus qu'à une opération univoque d'identification, elles invitent à un 
patient travail de discernement, qui est aussi une manière d'accomplir l'iter par lequel la vie terrestre peut mener jusqu'au salut ${ }^{69}$. De la même manière, on peut penser que les logiques relationnelles susceptibles de nouer entre eux les chapiteaux ne sont pas destinées à se donner d'emblée, mais doivent aussi faire l'objet d'un patient travail du sens. Retrouver sous le désordre des apparences le fil de l'iter ecclésial, en éprouver les détours avant de parvenir au but, pourrait bien constituer un mode de fonctionnement pertinent, parmi d'autres, du dispositif ecclésial. Enfin, ce que la méthode cartographique s'efforce de restituer n'est certes pas ce dont chaque fidèle était sommé de faire l'expérience. C'est l'écheveau maximal de relations qui ne se dévoile qu'à la mesure des capacités de discernement différenciées des utilisateurs du lieu et dont l'imperceptibilité relative contribue à doter le lieu rituel de sa charge d'énergie sacrale.

\section{Codage des cartographies}

Fig. 4

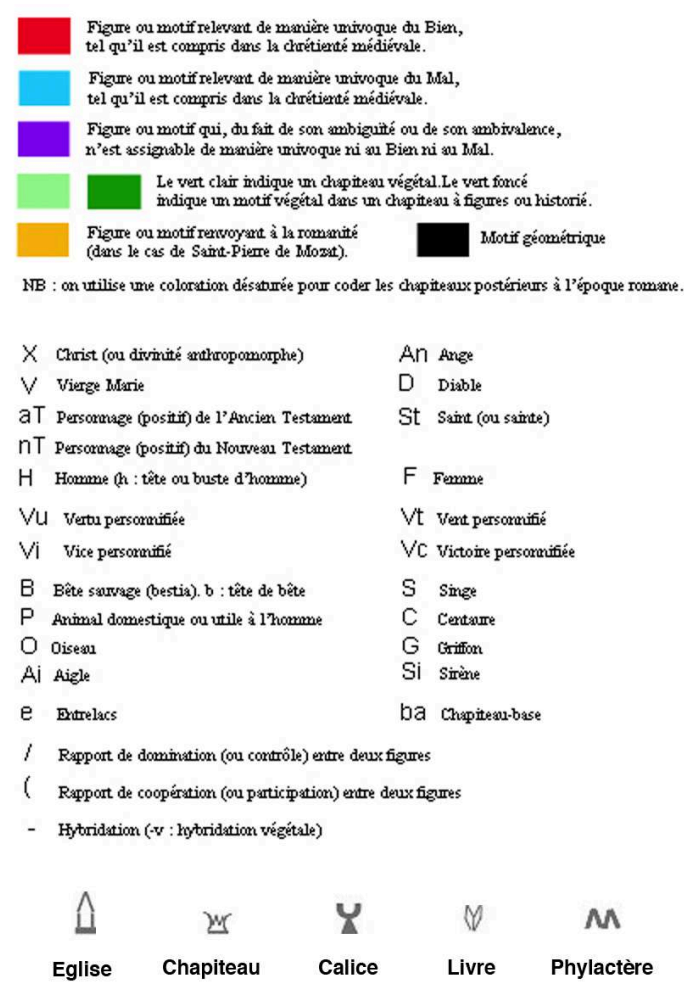

Couleurs, lettres et symboles utilisés dans le codage des cartographies

L'exercice cartographique, tel qu'on vient d'en définir les principes, suppose donc un codage aussi synthétique que possible de chaque chapiteau (ou face de chapiteau, dans les cas les plus complexes). Celui que l'on a élaboré associe un nombre limité de lettres (désignant des entités) et de couleurs (permettant de les qualifier). On commentera d'abord les lettres dont la figure 4 fournit la liste (ainsi que celle des symboles graphiques désignant des objets particulièrement importants : église ou autre construction sacrée, chapiteau, livre ou phylactère, calice). Si certains personnages sont identifiés par un codage spécifique, comme le Christ (X) ou la Vierge (V), les conceptions qui sous-tendent notre méthode cartographique conduisent à privilégier 
des codages génériques, car les relations entre les images deviennent beaucoup mieux perceptibles à ce niveau. Ainsi, toutes les figures (positives) de l'Ancien Testament ont été codées de la même façon (aT), de même que toutes celles du Nouveau (nT) ou que l'ensemble des saint(e)s non évangéliques (St). Tous les autres personnages humains ont été codés sans tenir compte de leur éventuelle identification spécifique (H ou F, pour les femmes) ${ }^{70}$. La même logique s'applique au monde animal. Certes, quelques espèces ont été singularisées, comme le singe (S), l'aigle (Ai) ou les créatures " merveilleuses ", particulièrement difficiles à classer, tels les centaures (C), les griffons (G) et les sirènes $(\mathrm{Si})$. Mais, pour le reste, nous avons opté pour les catégories génériques qui ordonnent les conceptions médiévales du monde animal (voir chapitre $\mathrm{VI}$ ). Ainsi, on distingue les bestiae (B), bêtes sauvages échappant au contrôle de l'homme, et le pecus $(\mathrm{P})$, c'est-à-dire les animaux domestiques qui restent soumis à son autorité, ou du moins qui lui sont utiles, comme les poissons. A ces deux grandes catégories, il convient d'ajouter les oiseaux, sans distinction d'espèces (0).

Par ailleurs, quand un chapiteau met en scène plusieurs figures différentes, le codage s'efforce d'exprimer les rapports élémentaires établis entre elles, en plaçant un signe entre les lettres qui leur correspondent. Ainsi, un rapport de domination ou de contrôle se marque par une barre oblique : par exemple, «H/S » transcrit le fait que l'homme tient le singe encordé. Un rapport de participation ou coopération est exprimé par une ligne courbe ouverte vers la figure qui bénéficie du soutien de l'autre : par exemple, « $\mathrm{P}(\mathrm{H}$ » indique que l'homme est porté par un animal qui lui vient en aide ou qu'il chevauche. L'hybridation est marquée par un tiret, notamment l'hybridation végétale ( «-V »). D'autres rapports, plus complexes, n'ont pu être exprimés graphiquement. Précisons enfin qu'il ne s'agit en aucune façon d'établir un codage de tous les éléments constitutifs d'un chapiteau mais plutôt de viser, dans la mesure du possible, la signification générique des principaux éléments et de leurs rapports ${ }^{71}$. Une même lettre peut valoir pour deux (voire trois) "individus" relevant du même codage. Sauf exception ${ }^{72}$, c'est seulement dans le cas des quaternités que l'on a pris le parti de dupliquer la lettre désignant l'entité concernée.

Outre le codage propre à chaque chapiteau, les cartographies s'emploient à faire apparaître les relations repérables entre plusieurs chapiteaux, proches ou éloignés. Sont ainsi indiqués les paires (trait plein) et les chapiteaux appariés, ou quasi-paires (trait pointillé), ainsi que les relations d'homologie ou d'association (trait à double flèche) et les relations d'opposition (trait à flèches affrontées). L'épaisseur des traits permet de distinguer des relations fortes et d'autres qui reposent sur des motifs partiels; leur couleur indique la tonalité dominante des éléments mis en relation.

Venons-en justement aux couleurs. On ne sera guère surpris que l'on distingue les figures ou motifs associés au Bien et ceux qui ont partie liée avec le Mal, dès lors que cette dualité joue un rôle majeur dans les représentations médiévales. Ainsi, le rouge code toute figure ou motif qui relève de manière univoque du Bien, tel qu'il est compris au sein de la chrétienté médiévale (soit tout ce qui émane de Dieu ou tend à s'en rapprocher). Cette couleur est celle de la divinité, des anges, des saints, des personnages positifs de l'Écriture, des figures et motifs liés à l'Ecclesia, ainsi que des personnifications des vertus. Certains animaux peuvent être codés en rouge, soit parce qu'ils appartiennent à une espèce dotée d'un prestige particulier (comme l'aigle), soit parce qu'ils sont figurés dans un contexte qui renvoie explicitement au sacré ecclésial ou à une moralisation positive. Ainsi, les griffons sont considérés comme positifs 
lorsqu'ils sont placés en paire symétrique de part et d'autre d'un calice, bien qu'ils puissent être négatifs dans d'autres cas. Quant aux figures humaines, elles sont codées en rouge quand elles manifestent qu'elles ont pris le parti du Bien, et se trouvent donc sur la voie du salut. Dans le chapiteau du singe cordé, l'homme est considéré comme une allégorie de la maitrise des passions animales et reçoit donc un tel codage.

Inversement, le bleu code toute figure ou motif qui relève de manière univoque du Mal, tel qu'il est compris au sein de la chrétienté médiévale (soit tout ce qui s'éloigne de Dieu). Reçoivent cette couleur les diables, les personnages bibliques ou historiques s'opposant aux desseins de Dieu et, dans le temps présent, les ennemis de l'Ecclesia et les hommes que le poids du péché entraîne vers la damnation. Reçoivent également cette couleur, les personnifications des vices, ainsi que certains animaux, soit parce qu'ils sont dotés d'une valeur univoquement maléfique (les dragons), soit parce qu'ils rentrent dans un rapport qui les qualifie comme tel (par un exemple, lorsqu'un griffon ou un lion agresse un personnage positif).

L'usage d'une troisième couleur, le violet, exige des explications particulières ${ }^{73}$. Il répond au souci de donner une place et une qualification propres à un très large ensemble de figures et de thèmes romans, qui ne se laissent pas répartir entre les catégories, théologiquement définies, du Bien et du Mal, soit en raison de leur ambivalence, soit parce qu'ils témoignent d'une relative indétermination. Faire place à cette catégorie constitue l'une des principales hypothèses qui sous-tendent notre approche. Elle nous semble d'autant plus importante que cette dimension des euvres romanes n'est généralement pas prise en considération dans les études iconographiques (ou que son statut y reste indéterminé).

Le violet, ainsi entendu, ne sert pas plus que les autres couleurs à désigner mais à qualifier certains états, voire certaines espèces d'êtres. Il s'agit d'abord de l'homme dans le temps présent de l'Ecclesia, marqué à la fois par les effets de la Chute et par les promesses de la Rédemption : il oscille entre la tentation du péché, qui le menace de perdition, et les moyens de salut à travers lesquels l'Église l'invite à bénéficier de la Grâce divine. Le violet qualifie donc l'état encore indéterminé de l'homme, dont on ne saurait dire s'il est voué à être sauvé ou damné. Plus largement, cette indétermination est le propre d'un monde mêlé, dans lequel les justes et les impies ne sont pas clairement identifiables. En effet, c'est seulement lors du Jugement dernier que la délimitation séparant les deux moitiés de l'humanité fera l'objet d'une pleine révélation. Jusque-là, prédomine au contraire la permixtio des deux cités, selon un terme employé par Augustin et repris jusqu'au XII ${ }^{\mathrm{e}}$ siècle au moins ${ }^{74}$. Cette notion de permixtio exprime avec force le statut du monde dans le temps présent de l'Église, un temps durant lequel les vérités restent voilées et les apparences trompeuses. Une telle conception semble bien constituer l'un des fondements de l'ambiguïté des représentations auxquelles nous confronte la sculpture romane. Bien que le combat du Bien et du Mal fasse rage, la ligne de partage entre eux n'est pas encore clairement identifiable. C'est ce caractère non encore déterminé, propre à un monde mélangé dans lequel l'homme demeure comme suspendu entre le Bien et le Mal, que vient notamment exprimer la couleur violette.

Mais le violet recouvre également une autre dimension. Il sert à qualifier le monde créé dans lequel vit l'homme. Ce monde est lui aussi marqué par la Chute : il est partiellement déchu, mais il ne cesse pas pour autant de témoigner de l'harmonie que le Créateur a instillé dans son œuvre. Ainsi, le violet peut qualifier les espèces végétales et animales, prises au sens littéral ou sur un mode qui allégorise les forces vitales de la 
création, mais aussi l'homme dont la vie est solidaire de toutes les forces cosmiques. Ce qui est mis en relief par le recours à cette couleur, c'est une certaine positivité du monde créé, qui n'est cependant pas susceptible d'être clairement ou univoquement référée au Bien, ce pourquoi on la qualifiera de positivité non morale. Positivité, parce que la création porte la trace de l'harmonie musicale que Dieu lui a conféré; non morale parce qu'elle échappe, pour l'essentiel, à la disjonction du salut et de la perdition. Dans le même temps, s'il est doté d'une valeur positive qu'on ne saurait ramener à la catégorie du Bien, le monde créé est aussi porteur de caractères inquiétants, et parfois menaçants pour l'homme, mais qu'on ne saurait pas davantage ramener nécessairement à la catégorie du Mal.

51 On a donc regroupé sous une même couleur catégorielle deux domaines distincts mais pas totalement coupés l'un de l'autre : le violet qualifie en effet, d'une part, un domaine intermédiaire entre le Bien et le Mal (et donc intérieur à leur opposition) et, d'autre part, un domaine extérieur ou irréductible à cette opposition. Intégrant tout ce qui est de l'ordre de l'ambiguïté ou de l'ambivalence, de l'indécidable propre à un monde mêlé ou encore d'une certaine positivité ou négativité non morales de la création, le violet couvre une ample gamme de motifs, allant des plus clairement positifs, mais non qualifiables en termes strictement moraux, jusqu'à de plus inquiétants, mais sans pour autant relever de façon explicite et univoque du Mal ou du diabolique. Les frontières étant particulièrement incertaines en de tels domaines, le choix du codage violet relève le plus souvent d'interprétations que nous argumenterons au fil de l'étude, mais qu'on pourra nous contester en tranchant dans un sens ou dans un autre l'équivoque constitutive des figures et motifs concernés.

Dans certains cas particulièrement délicats, on aura recours à des lettres bicolores, sans prétendre par là résoudre toutes les difficultés qu'implique la délimitation des usages des trois couleurs concernées. Néanmoins, on pourra ainsi exprimer soit un processus de conversion (mi-bleu mi-rouge), soit une orientation particulièrement marquée du violet vers le positif (mi-violet mi-rouge) ou vers le négatif (mi-violet mibleu).

53 Signalons enfin le recours à trois autres couleurs. Le vert est la couleur du végétal. Un rectangle vert clair indique un chapiteau à structure végétale (la moitié de ce rectangle indique que le chapiteau comporte seulement un registre végétal, au-dessus duquel prennent place des figures ou une scène historiée). Le vert foncé (associé à la lettre $\mathrm{V}$ ou v) est utilisé pour désigner un motif végétal jouant un rôle dans un chapiteau historié ou à figures. Le noir est la couleur des lettres désignant les éléments géométriques, comme les entrelacs (e) ou les chapiteaux-bases (ba).

Enfin, une couleur supplémentaire est utilisée dans le cas spécifique de Saint-Pierre de Mozat. Le jaune y caractérise les figures et motifs renvoyant de manière dominante à la romanité. Ce choix découle des hypothèses particulières que nous discuterons dans l'analyse du décor sculpté si singulier de Mozat; il sera donc explicité dans le chapitre II. Disons seulement que les figures ou thèmes faisant particulièrement référence à l'art antique sont codés par cette couleur (de même, une bordure jaune s'ajoute au rectangle vert clair des chapiteaux végétaux qui intensifient les citations des modèles corinthiens). Dans la mesure où les figures et motifs associés à la romanité échappent à la distinction du Bien et du Mal, en son sens chrétien, on peut considérer que la couleur jaune introduit une subdivision au sein du champ habituellement codé par le violet. De fait, bien des figures codées en jaune à Mozat apparaissent, dans d'autres édifices, qualifiées en violet (à l'exception notamment des aigles, que l'on rattache à la romanité dans le premier cas, aux valeurs spirituelles associées au Bien ailleurs). 

des frontières dont le tracé est nécessairement problématique; mais les controverses qui en découlent ne sauraient suffire à remettre en cause l'utilité de ladite typologie. En l'occurrence, le fait d'ajouter une catégorie intermédiaire (entre le Bien et le Mal) accentue les points d'hésitation et de débat, au lieu de les réduire. Les frontières sont bien souvent indécises entre ce qui relève d'une qualification positive ou négative et ce qui échappe au caractère tranchée de cette dichotomie. On pourra discuter tel ou tel de nos choix, mais nous soutenons qu'il est pertinent de faire droit à la catégorie symbolisée par le violet.

De manière générale, il est possible (sinon probable) que certaines décisions de codage aient pu infléchir indûment les analyses que nous tirons de nos cartographies. Mais l'essentiel, à nos yeux, est que celles-ci aident à faire apparaître des modalités d'agencement du décor qui ne sont peut-être pas absolument les seules, mais qui, sans elles, seraient restées hors de notre portée. Enfin, on peut soutenir que la difficulté du codage et les choix parfois frustrants auxquels il oblige constituent une dimension positive, créatrice, de la méthode cartographique. Le fait que l'on doive en permanence s'interroger pour déterminer si telle ou telle figure ou motif relève du Bien ou du Mal, demeure suspendu entre les deux ou bien participe d'une réalité placée comme en-deçà du Bien ou du Mal, participe sans doute du mode de fonctionnement, sinon du principe même du décor du lieu rituel. Celui-ci n'est-il pas, loin de tout programme définitivement clos sur ses certitudes, en quête de lui-même et de la difficile possibilité de mettre l'ordre-désordre du monde en résonance avec un ordre divin idéal ? N'invite t-il pas à un travail de discernement qui cherche à faire passer des apparences d'un monde mêlé au dévoilement d'une vérité, qui serait connaissance à la fois du Créateur et de sa création?

\section{NOTES}

1. Pour ces notions, voir Jérôme Baschet, L'iconographie médiévale, Paris, Gallimard, 2008, «Introduction : l'image-objet », p. 25-64.

2. Robert Fossier, Enfance de l'Europe. $\mathrm{XI}^{e}-\mathrm{XII}{ }^{e}$ siècles. Aspects économiques et sociaux, Paris, PUF, 2 vol., 1982. Sur la spatialisation des rapports sociaux, voir notamment Alain Guerreau, «Il significato dei luoghi nell'Occidente medievale : struttura e dinamica di uno 'spazio' specifico ", dans Enrico Castelnuovo et Giuseppe Sergi (éd.), Arti e Storia nel Medioevo. I. Tempi, Spazi, Istituzioni, Turin, Einaudi, 2002, p. 201-239 et "Stabilità, via, visione : le creature e il creatore nello spazio medievale », dans ibid., 2004, vol. III, p. 167-197, ainsi que Joseph Morsel, L'Histoire (du Moyen Age) est un sport de combat, Paris, LAMOP-Paris I, 2007, http://lamop.univ-paris1.fr/lamop/LAMOP/ JosephMorsel/index.htm.

3. Alain Guerreau, «Quelques caractères spécifiques de l'espace féodal européen », dans Neithard Bulst, Robert Descimon et Alain Guerreau (éd.), L'Etat ou le Roi. Les fondations de la modernité monarchique en France (XIV ${ }^{e}$-XVIII ${ }^{e}$ siècle), Paris, EHESS, 1996, p. 85-101.

4. Pour tout ce qui suit, Michel Lauwers, Naissance du cimetière. Espace sacré et terre des morts dans l'Occident médiéval, Paris, Aubier, 2005. Pour mieux souligner le rôle de l'église (et du cimetière)

Images Re-vues, Hors-série 3 | 2012 
dans la réorganisation spatiale de l'Occident, l'auteur a proposé de remplacer le terme d'encellulement par celui d'inecclesiamento.

5. Voir Dominique Iogna-Prat, La Maison Dieu. Une histoire monumentale de l'Église au Moyen Age, Paris, Seuil, 2006.

6. «He sunt manus, que, cum Deus omnium rerum sit creator, res in aliam transformant naturam, dum panis et vini substantiam in corpus transfundunt et sanguinem Iesu Christi (...). Ipsos lapides insensibiles in aliam transformant naturam, dum domum manufactam per consecrationem habitaculum faciunt trinitatis et angelorum ", Liber de vita christiana, II, 27, cité dans ibid., p. 415-416.

7. Dominique Iogna-Prat, Ordonner et exclure. Cluny et la société chrétienne face à l'hérésie, au judaïsme et à l'islam (1000-1150), Paris, Aubier, 1998.

8. Sur les métaphores du corps appliquées à l'église-édifice autant qu'à l'Eglise-communauté, voir M. Lauwers, Naissance, op. cit., p. 160 ; pour l'assimilation de la consécration au baptême, voir Bénédicte Palazzo-Bertholon et Éric Palazzo, «Archéologie et liturgie. L'exemple de la dédicace de l'église et de la consécration de l'autel », Bulletin monumental, 159/IV, 2001, p. 305-316.

9. Ibidem, p. 309 et pour l'assimilation de l'église au saint, qui en fonde l'ancrage local, voir A. Guerreau, « Il significato dei luoghi », op. cit.

10. Voir Didier Méhu (dir.), Mises en scènes et mémoires de la consécration de l'église dans l'occident médiéval, Turnhout, Brepols, 2009.

11. Sur le lien entre l'extension du décor extérieur et l'intensification du rôle de l'église dans la polarisation de l'espace social (c'est-à-dire aussi entre la mise en place du décor de façade et la constitution de l'enveloppe sacrale de l'église), voir Alessia Trivellone, «Le développement du décor monumental et la conquête de l'extérieur des églises : sagreres et façades catalanes au cours de la première moitié du XI ${ }^{\mathrm{e}}$ siècle ", dans Michel Lauwers (éd.), Lieux sacrés et espace ecclésial (IXeXVe siècles), Cahiers de Fanjeaux, 46, 2011, p. 175-227.

12. Caroline Roux a bien analysé la fréquence de la localisation de l'accès principal sur le flanc de la nef (principalement le flanc sud) dans les églises d'Auvergne et de nombreuses régions de la France méridionale (La pierre et le seuil. Portails romans en Haute-Auvergne, Clermont-Ferrand, Presses Universitaires Blaise-Pascal, 2004, p. 79-103).

13. Rappelons l'importante observation d'Alain Guerreau : «Tout édifice était considéré comme l'enveloppe délimitant, protégeant, configurant un espace intérieur» («Édifices médiévaux, métrologie, organisation de l'espace. A propos de la cathédrale de Beauvais ", Annales E.S.C., 47, 1992, p. 87-106; ainsi que « Post-scriptum. Mensura, représentation du monde, structures sociales ", Histoire \& mesure, 16-3/4, 2001; http://histoiremesure.revues.org/126).

14. Sur le rite de dédicace, voir D. Iogna-Prat, La Maison Dieu, op. cit., p. 266sq. et Didier Méhu, " Historiae et Imagines de la consécration de l'église au Moyen Age », dans D. Méhu (dir.), Mises en scènes, op. cit., p. 15-48.

15. "Quod Ecclesia intus ornatur festive, non extra, moraliter innuit quod omnis gloria eius ab intus est ", Manuale de mysteriis ecclesiae, éd. Marie-Thérèse d'Alverny, "Les Mystères de l'église d'après Pierre de Roissy », dans Pierre Galais et Yves-Jean Riou (éd.), Mélanges offerts à René Crozet, Poitiers, 1966, p. 1096-1097.

16. Voir notamment Jean Hubert, «La place faite aux laïcs dans les églises monastiques et les cathédrales aux $\mathrm{XI}^{\mathrm{e}}$ et $\mathrm{XII}^{\mathrm{e}}$ siècles ", repris dans Arts et vie sociale de la fin du monde antique au Moyen Age, Genève, Droz, 1977, p. 470-487 ; Arnold W. Klukas, « The Architectural Implications of the Decreta Lanfranci ", dans Reginald A. Brown (éd.), VI Proceedings of the Battle Conference on Anglo-Norman Studies, Woodbridge, The Boydell Press, 1984, p. 136-171; Thomas Creissen, «Les clôtures de chœur des églises d'Italie à l'époque romane : état de la question et perspectives ", Hortus Artium Medievalium, 5, 1999, p. 169-181 ; Jacqueline Jung, «Beyond the Barrier : The Unifying Role of the Choir Screen in Gothic Churches", The Art Bulletin, 82, 2000, p. 622-657; Paolo Piva, «Lo 'spazio liturgico' : architettura, arredo, iconografia (secoli IV-XII) », dans Paolo 
Piva (dir.), L'arte medievale nel contesto (300-1300). Funzioni, iconografia, tecniche, Milan, Jaca Book, 2006, p. 141-180.

17. C'est ce que précise une attestation précoce de cette séparation, dans le contexte de la Pataria milanaise (P. Piva, ibid., p. 155). Voir les dispositifs restitués par A. Klukas (ibid.) et l'imposant mur de chœur de l'ancienne cathédrale de Nice, vers 1049 (Jacques Thirion, «L'ancienne cathédrale de Nice et sa clôture de chœur du $\mathrm{XI}^{\mathrm{e}}$ siècle d'après les découvertes récentes ", Cahiers archéologiques, 17, 1967, p. 120-160).

18. Pour tout ceci, voir Jérôme Baschet, La civilisation féodale. De l'an mil à la colonisation de l'Amérique, Paris, Champs-Flammarion, 2006, chapitre 3.

19. Par exemple Honorius Augustodunensis, De gemma animae, I, 126, PL, 172, c. 584 ; Sicard de Crémone, Mitrale, I, 1, PL, 213, c. 15.

20. P. Piva, «Lo 'spazio liturgico' », op. cit., ainsi que Marie-Thérèse Camus et Claude AndraultSchmitt (dir.), Notre-Dame la Grande de Poitiers. L'œuvre romane, Paris-Poitiers, Picard-CESCM, 2002, p. 160-186 et Cécile Treffort, «Mémoires de chœurs. Monuments funéraires, inscriptions mémorielles et cérémonies commémoratives à l'époque romane ", dans Claude Arrignon, MarieHélène Debiès, Claudio Galderisi et Éric Palazzo (éd.), Cinquante années d'études médiévales. A la confluence de nos disciplines, Turnhout, Brepols, 2005, p. 219-232. Sur l'importance de l'arc triomphal, voir les recherches de Caroline Roux, notamment «A propos de l'arc triomphal. Origine, formes et emplacements dans l'espace ecclésial (IV ${ }^{\mathrm{e}}$ XII ${ }^{\mathrm{e}}$ siècle) », dans Anne Baud (éd.), Espace ecclésial et liturgie au Moyen Age, Lyon, Maison de l'Orient et de la Méditerranée, 2010, p. 153-181.

21. Il distingue corpus, cancellus et sanctuarium (Rationale divinorum officiorum, I, 1, 14, éd. citée, p. 17). L'articulation entre dualité et ternarité est du reste un trait structurel des représentations médiévales.

22. Quelques précisions de vocabulaire peuvent être utiles. On distinguera la nef au sens architectural (de la façade au transept) et la nef au sens liturgique (espace propre aux laïcs), puisque, le plus souvent, une partie de la nef architecturale est occupée par le chœur liturgique. On emploiera le terme "chœur» uniquement dans son sens liturgique (en récusant l'usage commun qui l'utilise pour désigner le lieu de l'autel majeur). Si on privilégie ici le terme liturgique "sanctuaire ", on peut aussi nommer "chevet » l'ensemble de la partie orientale de l'église (sanctuaire, déambulatoire et absidioles). Notons du reste que l'un des termes latins désignant le «chevet » au Moyen Age est caput, renvoyant ainsi à l'homologie entre l'église et le corps humain. Au-delà des conventions du vocabulaire actuel, il est essentiel de s'interroger sur les termes utilisés au Moyen Age et sur la conception de l'édifice qu'ils engagent. Voir, dans cette perspective, l'étude de $\mathrm{D}$. Méhu, « Les figures de l'édifice ecclésial d'après le 'Guide du pèlerin de Saint-Jacques de Compostelle' ", dans Michel Lauwers (éd.), Lieux sacrés et espace ecclésial (IXe-XVe siècles), Cahiers de Fanjeaux, 46, 2011, p. 79-113.

23. La présence, à Notre-Dame-du-Port (fig. 2) et à Orcival notamment, de deux demi-colonnes montantes mais ne supportant aucun arc - et alors que les autres piles de la nef en sont dépourvues -, renvoie très certainement à un dispositif liturgique spécifique, soit que ces colonnes supportaient une "poutre de gloire" à laquelle pouvait être suspendu un crucifix associé à l'autel de la croix, soit qu'elles visaient à marquer architecturalement une délimitation liturgique. Dans d'autres régions, certaines particularités dans le traitement des colonnes de la nef ont pu être mises en relation avec la distinction entre chœur liturgique et nef des laïcs ou encore avec la position de l'autel de la croix; voir Eric Fernie, "The Use of Nave Supports in Romanesque and Early Gothic Churches ", Gesta, 23, 1984, p. 107-117 (notamment p. 113, où la présence d'un crucifix de bois est attesté « in medio ecclesiae », c'est-à-dire sans doute en rapport avec l'autel de la croix, dans la cathédrale de Laon à la fin du XII ${ }^{\mathrm{e}}$ siècle) et, du même, " L'espace liturgique dans les édifices de l'Angleterre normande ", dans Cinquante années d'études médiévales, op. cit., p. 191-200). 
24. Pour la critique de l'interprétation classique du déambulatoire, voir notamment Beat Brenk, "Les églises de pèlerinage et le concept de prétention », dans Nicolas Bock, Peter Kurmann, Serena Romano et Jean-Marie Spieser, Art, Cérémonial et Liturgie au Moyen Age, Rome, Viella, 2002, p. 127-136 et Paolo Piva, "Le déambulatoire et les 'trajets' de pèlerinage dans les églises d'Occident ( $\mathrm{X}^{\mathrm{e}}-\mathrm{XII}{ }^{\mathrm{e}}$ siècle), dans Paolo Piva (éd.), Art médiéval. Les voies de l'espace liturgique, Paris, Picard, 2010, p. 80-129. Voir également Pierre Martin, «Autour du chœur, le déambulatoire. Terminologie et historiographie ", dans Cinquante années d'études médiévales, op. cit., p. 241-250 et Éric Sparhubert, "Sur la route de Compostelle : le chevet d'une "église de pèlerinage " à l'épreuve de la liturgie, Saint-Léonard de Noblat ", dans Éric Sparhubert et Cécile Voyer (éd.), L'image médiévale: Fonctions dans l'espace sacré et structuration de l'espace cultuel, Turnhout, Brepols, 2011, p. 41-68. Nous remercions Éric Sparhubert pour les précieuses indications qu'il nous a fournies sur ces questions.

25. On le verra dans le cas de Saint-Nectaire, au chapitre III.

26. Nous n'évoquons ici qu'une situation spécifique, parmi d'autres possibles : la gamme va de l'inaccessibilité à peu près complète des reliques à un accès très direct (voir l'étude de P. Piva, citée dans la note 24). Ajoutons que les reliquaires pouvaient, lors de certaines occasions festives, être exhibés hors de leur lieu habituel, voire portés en procession.

27. C'est le binôme caput/corona qui semble ici pertinent (voir D. Méhu, «Les figures ", op. cit.). Par ailleurs, on admettra que les tribunes des bas-côtés ne faisaient l'objet que d'un accès très restreint (c'est sans doute par exception que certains témoignages, comme à Conques, évoquent la présence des pèlerins dans cette partie de l'édifice). L'existence des tribunes peut se comprendre avant tout comme une option architectonique et symbolique (voir la synthèse récente de P. Piva, ibid.), sans pour autant négliger le fait que, au moins dans certaines occasions, le chœur des chanteurs pouvait prendre place dans la travée la plus orientale des tribunes (qui, dans les églises d'Auvergne, est souvent distinguée par une arcature plus étroite). Quant à l'étage du narthex, il est souvent associé à des fonctions liturgiques spécifiques (rituel pascal, liturgie funéraire, etc.).

28. Jean-Pierre Caillet, «Architecture et décor monumental », dans Pierre Riché (dir.), L'Europe de l'an mil, La-Pierre-qui-Vire, Zodiaque, 2001, p. 77-255.

29. Voir Christian Sapin (dir.), Avant-nefs et espaces d'accueil dans l'église entre le IV et le XII siècle, Paris, CTHS, 2002.

30. C'est ce dispositif liturgique, bien attesté dans les édifices anglo-normands réformés par Lanfranc de Cantorbery (cf. A. Klukas, "The Architectural», op. cit.), que l'on s'efforcera de restituer pour les édifices auvergnats étudiés ici.

31. On notera que, de manière générale, les clercs des XII ${ }^{\mathrm{e}}-\mathrm{XIII}{ }^{\mathrm{e}}$ siècles accordent une prééminence à la longueur par rapport aux autres dimensions (largeur et hauteur); voir sur ce point les remarques d'Alain Guerreau, « Post-scriptum », op. cit.

32. "longitudo eius longanimitas est quae patienter adversa tolerat donec ad patriam perveniat ", Mitrale, I, 4, éd. citée, col. 20.

33. Voir A. Guerreau, "Le champ sémantique de l'espace dans la Vita de saint Maieul (Cluny, début du XI ${ }^{\mathrm{e}}$ siècle) », Journal des Savants, 1997, p. 363-419 : les deux termes les plus usités sont iter et via.

34. Cette conjonction contradictoire contribue sans doute, par l'écart qu'elle marque avec l'univers commun, à la sacralité de l'église. Elle relève aussi du « hors espace ", lieux retranchés de l'espace et, pour cela, points de passage entre les hommes et Dieu; cf. A. Guerreau, «Quelques caractères ", op. cit., p. 96-99.

35. Il s'agit d'un emprunt à Gn 28, 17 ; voir M. Lauwers, Naissance, op. cit., p. 67. De manière similaire, Augustin décrit le Tabernacle comme un locus clos et protégé, mais suggère en même temps que le parcours de ses différentes parties est un cheminement vers Dieu; cf. Mary 
Carruthers, Machina Memorialis. Méditation, rhétorique et fabrication des images au Moyen Age, Paris, Gallimard, 2002, p. 315-317.

36. Par exemple à Saint-Pé de Bigorre : «Est domus hic domini, via caeli »; cité par Robert Favreau "Le thème épigraphique de la porte ", dans La façade romane, Cahiers de Civilisation médiévale, 34, 1991, p. 269-270.

37. D. Méhu, «Locus, transitus, peregrinatio. Remarques sur la spatialité des rapports sociaux dans l'Occident médiéval (XI ${ }^{\mathrm{e}}$-XIII ${ }^{\mathrm{e}}$ siècle) », dans Construction de l'espace au Moyen Age: pratiques et représentations, Paris, Publications de la Sorbonne, 2007, p. 275-293.

38. Sur la présence, à l'époque romane, de la Maiestas Domini à proximité de l'autel et en rapport avec la célébration eucharistique, voir Piotr Skubiszewski, "Maiestas Domini et liturgie », dans Cinquante années d'études médiévales, op. cit., p. 309-408.

39. Barbara Franzé, «Une lecture en contexte : les peintures de l'église Saint-Nicolas de Tavant », Hortus Artium medievalium, 13/2, 2007, p. 471-490.

40. Staale Sinding-Larsen, Iconography and Ritual. A Study of Analytical Perspectives, Oslo, Universitetsforlaget, 1984, p. 17-25 et 96-97.

41. Dominique Allios, "Saint-Nectaire en Auvergne : de l'invention à la glorification d'une mémoire », dans Arturo Carlo Quintavalle (dir.), Medioevo: immagine e memoria, Milan, Electra, 2009, p. 194-204.

42. Voir ibidem. A Issoire, des traces de faux appareil peint sont conservées dans le bas-côté sud de la nef (elles ont pu servir d'inspiration pour la « restitution » du décor peint au XIX ${ }^{\mathrm{e}}$ siècle).

43. Voir notamment Hans Peter Autenrieth, «Structures ornementales et ornements à motifs structuraux : les appareils peints jusqu'à l'époque romane ", dans Le rôle de l'ornement dans la peinture murale du Moyen Age, Colloque de Saint-Lizier (Collection Civilisation médiévale, IV), Poitiers, CESCM, 1997, p. 57-72. La polychromie architecturale peut aussi contribuer à distinguer les différents espaces liturgiques (voir Géraldine Victoir, «La polychromie de la cathédrale de Noyon et la datation des voûtes quadripartites de la nef », Bulletin monumental, 163/III, 2005, p. 251-254).

44. De ce point de vue, l'essor des chapiteaux sculptés devrait être analysé dans le cadre d'un processus plus large d'expansivité des images-objets tridimensionnelles (Majestés et statues d'autel, décor sculpté intérieur puis extérieur).

45. Surtout si l'on prend en compte les chapiteaux réalisés en stuc. Voir Le stuc, visage oublié de l'art médiéval (Exposition du Musée Sainte-Croix de Poitiers), Paris-Poitiers, Somogy-Musées de Poitiers, 2004.

46. J.-P. Caillet, « Architecture », op. cit., p. 236-239.

47. Victor Lassalle l'a exprimé de manière particulièrement claire : "L'art médiéval (jusqu'à l'époque romane) a substitué à la conception antique d'un décor sculpté fait d'éléments répétitifs une diversification systématique de ces éléments» («Quelques exemples de répartition ordonnée des chapiteaux ornementaux des $\mathrm{XI}^{\mathrm{e}}$ et $\mathrm{XII}^{\mathrm{e}}$ siècles dans les églises de l'est et du sud de la France ", Revue d'Auvergne, 562, 2002, p. 135-157).

48. Il s'agit là de tendances générales, et il ne faut pas oublier que l'essor de l'architecture gothique n'exclut pas le maintien, fort durable dans certaines régions, des principes constructifs romans.

49. Voir Eliane Vergnolle, «La colonne à l'époque romane. Réminiscences et nouveautés", Cahiers de Civilisation médiévale, 41, 1998, p. 141-174, ainsi que John Onians, Bearers of Meanings. The Classical Orders in Antiquity, the Middle Ages and the Renaissance, Princeton, Princeton UP, 1988.

50. Voir Leah Rutchick, "Visual Memory and Historiated Sculpture in the Moissac Cloister ", dans Peter Klein (éd.), Die mittelalterliche Kreuzgang. The Medieval Cloisters. Le cloître du Moyen Age. Architektur, Funktion und Programm, Munich, Schnell-Steiner, 2003, p. 190-211.

51. "In omnibusque columnarum capitibus sanctorum reliquias diligenter induci iussit", MGH, Scriptores, NS, IX, éd. R. Holtzmann, Berlin, 1935, p. 58; voir Jean-Pierre Caillet, « Reliques et 
architecture religieuse aux époques carolingienne et romane", dans Les reliques : Objets, cultes, symboles (Colloque international de l'Université du Littoral-Côte d'Opale), Turnhout, Brepols, 1999, p. 169-197, ainsi que D. Iogna-Prat, La Maison Dieu, op. cit., p. 544-545.

52. Ibidem.

53. Cité et commenté par Elisabeth Declercq, « La place des reliques dans le mobilier liturgique et l'architecture d'après les Gesta Karoli Magni de l'abbaye de Lagrasse ", Cahiers de Saint-Michel de Cuxa, 14, 1983, sans pagination.

54. Voir les cas rapportés par E. Declercq, ibid. Elle mentionne également que l'autel de l'église Saint-Just de Valcabrède contenait un chapiteau pré-roman déposé, dans la cavité duquel des reliques, scellées par une plaque de marbre, étaient accompagnées d'un parchemin d'authentification daté de 1200.

55. Ailleurs, on en place dans les murs, comme à l'abbatiale de Maillezais (mentionné par D. Iogna-Prat, op. cit., p. 545).

56. L'ambiguïté demeure en ce qui concerne l'expression "caput columnae » : elle désigne sans doute le plus souvent le sommet de la colonne elle-même; mais il n'est pas exclu qu'elle puisse désigner le chapiteau. Il semble que ce soit le cas par exemple dans les Gesta de Gérard II de Cambrai (1076-1092) : "capita columnarum in utroque latere turpiter fixa et corrupta decenter coronavit", cité dans Victor Mortet et Paul Deschamps, Recueil de textes relatifs à l'histoire de l'architecture et de la condition des architectes du Moyen Age, Paris, 1911, vol. I, n. 68.

57. Bède le Vénérable, De Templo Salomonis, ch. 17, PL, 91, col. 779 et Raban Maur, De Universo, ch. 23, PL, 111, col. 404.

58. Les commentaires liturgiques sur l'édifice ecclésial et ses parties peuvent être considérés dans leur entier comme une manière de spiritualiser le bâtiment. Cette spiritualisation est pour l'essentiel une cléricalisation : presque toutes les parties de l'édifice sont assimilées à des figures cléricales, à l'exception du pavement (rapproché du vulgus), des tuiles (rapprochées des milites), ainsi que des pierres des murs (assimilées à l'ensemble des fidèles, liés par le ciment de la caritas). 59. Isidore de Séville indique déjà que les colonnes "supportent le poids de toute la structure » (Et., XV, 8, 13). Et J. Onians (Bearers of Meanings, op. cit., p. 75) de noter que cette idée de support de la charge de l'édifice est nouvelle et ne correspond pas à la fonction que l'art antique attribue aux colonnes.

60. Voir par exemple J.-P. Caillet, "Architecture ", op. cit., p. 238 et 253 et Beat Brenk, "Originalitá e innovazione nell'arte medievale », dans Enrico Castelnuovo et Giuseppe Sergi (éd.), Arti e Storia nel Medioevo. I, op. cit., p. 58-60. L'un et l'autre associent le statut de cette zone intermédiaire à des types de représentation spécifiques (que B. Brenk définit de manière peu appropriée comme «fantaisies » de l'artiste, et que J.-P. Caillet inscrit dans le spectre large allant des «menaces » pesant sur les pécheurs aux interventions divines pour l'en délivrer). C'est sans doute André Scobeltzine qui a le plus développé l'analyse des chapiteaux comme «espace de transition ", à la fois jonction et passage. Il associe ce statut moins à des thématiques représentationnelles spécifiques qu'au constat d'un "foisonnement de vie » et d'une densité visuelle particulièrement intenses. Il souligne néanmoins que les chapiteaux jouent souvent comme expression du conflit entre le bien et le mal, ou comme espace d'indécision entre ces deux pôles (L'art féodal et son enjeu social, Paris, Gallimard, 1973, p. 137sq.).

61. Les chapiteaux placés en paire symétrique sur l'arc triomphal en soulignent la valeur de passage. Ceux de l'arc triomphal de l'église de Champeix (Puy-de-Dôme) portent les inscriptions des noms donnés aux colonnes du Temple (Iaquin et Boaz), ce qui en accentue encore la valorisation (voir C. Roux, « A propos de l'arc triomphal », op. cit.).

62. Pour une discussion de ces conceptions, voir J. Baschet, L'iconographie médiévale, op. cit., ch. 4.

63. Pour un usage caricatural de cette notion, voir William Travis, « The Iconography of the Choir Capitals of Saint-Lazare of Autun and the anagogical way in Romanesque Sculpture", Konsthistorisk Tidskrift/Journal of Art History, 68, 1999, p. 220-249, qui se situe précisément dans 
l'alternative que l'on récuse ici : critiquant les auteurs pour qui les chapiteaux sont disposés au hasard, il affirme qu'ils forment un programme dont il s'agit de découvrir le «message ». Plus généralement, et notamment pour les essais récents de renouvellement, voir Jean-Marie Guillouët et Claudia Rabel (éd.), Le Programme. Une notion pertinente en histoire de l'art médiéval?, Paris, Le Léopard d'or, 2011). On peut également se référer à l'étude, spécifiquement consacrée au cas des chapiteaux, de Marcello Angheben (Les chapiteaux romans de Bourgogne. Thèmes et programmes, Turnhout, Brepols, 2003, notamment p. 13 et 449-454). D'un côté, le terme "programme » y est volontairement vidé de toute signification précise et désigne simplement l'ensemble des chapiteaux d'un édifice donné (en même temps, la question de l'intentionalité, dont on doit mesurer à quel point elle est attachée à la notion de programme, fait subrepticement retour, en dépit de l'extrême neutralité de la définition adoptée). De l'autre, est proposée la notion de "programme iconographique", dans laquelle se trouvent noués deux critères : « cohérence » et « réflexion préalable ». C'est sur la conception de ces deux notions (et sur leur lien supposé) que portent les réserves formulées ici (sans parler de la dissociation entre ce qui relève de l'iconographie et le « reste ", sur laquelle on revient plus loin).

64. Pour une analyse de la dimension ornementale de l'art médiéval, voir les travaux de JeanClaude Bonne, en particulier « De l'ornemental dans l'art médiéval (VII ${ }^{\mathrm{e}}-\mathrm{XII}{ }^{\mathrm{e}}$ siècle). Le modèle insulaire ", dans Jérôme Baschet et Jean-Claude Schmitt (éd.), L'image. Fonctions et usages des images dans l'Occident médiéval, Cahiers du Léopard d'Or, 5, Paris, Léopard d'or, 1996, p. 207-249.

65. Bien entendu, la continuité formelle des ensembles peints ne présuppose pas des rapports simples, de type uniquement narratifs; voir à ce sujet Jérôme Baschet, Lieu sacré, lieu d'images. Les fresques de Bominaco (Abruzzes, 1263). Thèmes, parcours, fonctions, Paris-Rome, La Découverte-EFR, 1991.

66. V. Lassalle (« Répartition ordonnée », op. cit.) a bien souligné cette volonté d'éviter la rupture de l'unité du lieu que provoquerait la constitution de sous-ensembles d'une cohérence telle qu'ils se refermeraient sur eux-mêmes. D'où le recours à des séries évolutives et le maintien d'un certain enchevêtrement propice à faire jouer l'unité (différenciée) du lieu ecclésial.

67. Pour une approche de la pluralité des niveaux de signification, et en particulier pour la notion de sens générique, voir L'iconographie médiévale, op. cit., ch. 4 et 5.

68. L'étude de Victor Lassalle («Quelques exemples», op. cit.) est l'une de celles qui abordent ces questions de la façon la plus convaincante. L'auteur parvient notamment à mettre en évidence une distribution cohérente des différents types de chapiteaux ornementaux, notamment dans l'abbatiale de Sylvacane : dans un contexte cistercien, la distribution procède du plus orné, autour du portail, au plus austère, dans l'abside. Le livre de M. Angheben (Les chapiteaux romans, op. cit.) comporte des apports notables (voir aussi sa contribution «Sculpture romane et liturgie ", dans Paolo Piva (éd.), Art médiéval, op. cit., p. 131-179). Prenant judicieusement appui sur des niveaux de sens génériques, il met en évidence, dans un nombre significatif d'édifices, une différenciation tendancielle entre les chapiteaux de la nef et ceux du sanctuaire. Les premiers accordent une large place au paradigme du combat spirituel, exprimant la position de l'Église menacée par le mal, tandis que les seconds font contraste par la fréquence des thèmes et des valeurs formelles évoquant la paix et l'harmonie paradisiaques. Il a également remarqué, dans les édifices bourguignons, une opposition fréquente entre la disposition symétrique des paires de chapiteaux placées dans le sanctuaire et leur disposition non symétrique dans les autres parties de l'édifice. Toutefois, tous les types de rapports constitutifs de l'agencement des chapiteaux sont loin d'être pris en compte et l'auteur écarte de l'analyse les chapiteaux végétaux. Ce faisant, il s'interdit toute saisie de la seule unité d'analyse pertinente : la totalité du décor sculpté et son articulation avec le lieu rituel, perçu dans sa globalité.

69. Cette notion a été judicieusement esquissée par Thierry Lesieur, Devenir fou pour être sage. Construction d'une raison chrétienne à l'aube de la réforme grégorienne, Turnhout, Brepols, 2003. Sur le rôle de l'énigme dans l'art roman, voir aussi Anne-Marie Bouché, «Vox imaginis: Anomaly and 
Enigma in Romanesque Art ", dans Anne-Marie Bouché et Jeffrey Hamburger (éd.), The Mind's Eyes. Art and Theological Argument in the Middle Age, Princeton UP, 2006, p. 306-335.

70. On identifie aussi des figures spirituelles (ange : An; diable : D) et des personnifications (vertu : Vu; vice : Vc; Victoire : Vi; Vent: Vt).

71. Le travail de codage diffère considérablement de l'indexation iconographique, telle qu'on peut la pratiquer afin de constituer une base de données. L'extrême concentration de l'information est ici étroitement liée aux contraintes d'une restitution cartographique de l'ensemble du décor de l'édifice.

72. Dans le cas de Chanteuges, on a dupliqué la même lettre lorsque deux figures identiques sont disposées symétriquement de part et d'autre de l'axe médian du chapiteau, parce qu'il a semblé pertinent, dans le cas de cet édifice, de souligner un rapprochement entre les chapiteaux présentant ce type de construction.

73. Précisons que le choix du rouge et du bleu est purement arbitraire. Il ne cherche évidemment en rien à évoquer un quelconque symbolisme médiéval des couleurs (on nous a même reproché de prendre celui-ci à revers). La seule justification, pour le choix du rouge et du bleu, est qu'il nous permet d'évoquer chromatiquement, le caractère " intermédiaire » de la troisième couleur, le violet.

74. Sur cette notion, voir Elisa Brilli, Una vicina città. Storia del paradigma della civitas diaboli nel Occidente medievale, thèse de doctorat sous la direction de Jean-Claude Schmitt, EHESS-Université de Rome-I, 2009, ch. I, notamment p. 21 sq. Outre Augustin lui-même, E. Brilli cite Otton de Freising qui, au XII ${ }^{\mathrm{e}}$ siècle, qualifie l'Église de « civitas permixta ».

\section{INDEX}

Keywords : romanesque art, capitals, Auvergne, Middle ages, anthropology, iconography, church, liturgy, soul, body

Mots-clés : art roman, chapiteaux, Auvergne, Moyen Age, anthropologie, iconographie, église, liturgie, rituel, âme, corps

\section{AUTEURS}

\section{JÉRÔME BASCHET}

EHESS, GAHOM 